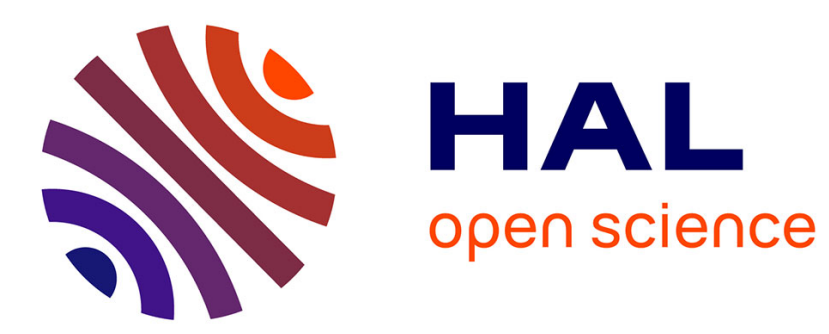

\title{
Equilibrium dynamics in an aggregative model of capital accumulation with heterogeneous agents and elastic labor
}

\author{
Cuong Le Van, Manh-Hung Nguyen, Yiannis Vailakis
}

\section{To cite this version:}

Cuong Le Van, Manh-Hung Nguyen, Yiannis Vailakis. Equilibrium dynamics in an aggregative model of capital accumulation with heterogeneous agents and elastic labor. Journal of Mathematical Economics, 2007, 43 (3-4), pp.287-317. 10.1016/j.jmateco.2006.06.003 . halshs-00101237

\section{HAL Id: halshs-00101237 \\ https://shs.hal.science/halshs-00101237}

Submitted on 26 Sep 2006

HAL is a multi-disciplinary open access archive for the deposit and dissemination of scientific research documents, whether they are published or not. The documents may come from teaching and research institutions in France or abroad, or from public or private research centers.
L'archive ouverte pluridisciplinaire HAL, est destinée au dépôt et à la diffusion de documents scientifiques de niveau recherche, publiés ou non, émanant des établissements d'enseignement et de recherche français ou étrangers, des laboratoires publics ou privés. 


\title{
Equilibrium dynamics in an aggregative model of capital accumulation with heterogeneous agents and elastic labor
}

\author{
Cuong Le Van, Manh-Hung Nguyen and Yiannis Vailakis* \\ Centre d' Economie de la Sorbonne, Université Paris-1, Pantheon-Sorbonne, CNRS \\ 106-112 Bd de l' Hôpital, 75647 Paris Cedex 13, France
}

June 2006

\begin{abstract}
The paper extends the canonical representative agent Ramsey model to include heterogeneous agents and elastic labor supply. The welfare maximization problem is analyzed and shown to be equivalent to a non-stationary reduced form model. An iterative procedure is exploited to prove the supermodularity of the indirect utility function. Supermodularity is subsequently used to establish the convergence of optimal paths.
\end{abstract}

Keywords: Single-sector growth model, heterogeneous agents, elastic labor supply, supermodularity

JEL Classification: C62, D51, E13

${ }^{*}$ We are grateful to an anonymous referee for valuable comments and suggestions. ManhHung Nguyen acknowledges financial support from the Vietnam Ministry of Education (Project 322). Yiannis Vailakis acknowledges the financial support of a Marie Curie fellowship (FP6 Intra-European Marie Curie fellowships 2004-2006).E-mail: levan@univ-paris1.fr, manh-hung.nguyen@malix.univ-paris1.fr, vailakis@univ-paris1.fr. Correspondence: Cuong Le Van 


\section{Introduction}

Optimal growth theory is useful in qualitatively characterizing simple dynamical systems and in providing constructive methods for the quantitative analysis of the solutions to more complex ones. The usefulness is, for some purposes, enhanced because of the intimate connections between optimal growth theories and their equilibrium counterparts. In a decentralized economy, we seek knowledge about the time paths of the various prices for goods and production factors as well as the distribution of income and wealth. Dynamic optimization techniques used extensively in growth theory facilitate the study of the evolution of those economic aggregates.

A major concern in the area of optimal growth has been the analysis of the short-run and asymptotic behavior of optimal solutions. At issue are questions concerning the existence and asymptotic stability of optimal programs with respect to the stationary optimal stock (turnpike results) as well as the possibility of cyclical or even chaotic behavior.

One-sector representative agent models where utility is derived solely from consumption have been studied extensively in the literature under a variety of different technological specifications. A well known property of those models is the monotonicity of the optimal capital path. This property is persistent even when the technology exhibits increasing returns and investment is irreversible (see Dechert and Nishimura (1983), Dimaria, Le Van and Morhaim (2002), Duran and Le Van (2003)). Thus, it is often suggested that one-sector models display simple dynamics.

Becker and Foias (1987) show that agents' heterogeneity plays a crucial role to the appearance of nonmonotonic dynamics in a single-sector model. Studying a specific economy with incomplete markets as represented by borrowing constraints, they demonstrate that deterministic cycles of period 2 may occur. In Becker and Foias (1994), they discuss in more detail the issue of equilibrium cycles and their construction using bifurcation analysis. Their work has been further elaborated by Sorger (1994).

In a different setting (i.e complete market structure), Le Van and Vailakis (2003) have also shown that the monotonicity property does not carry over if one permits many consumers with different discount factors. The model does not exhibit cyclical behavior. The convergence of the optimal capital sequence to a particular stock $k^{s}$ is still true, but that stock is not itself a steady state ${ }^{1}$. This result implies that the optimal capital sequence initiated at $k_{0}=k^{s}$ converges to $k^{s}$ in the long-run, but it is not a constant sequence. Hence, the resulting optimal capital path cannot be monotonic. The model

\footnotetext{
${ }^{1}$ An early turnpike result for multisector economies with heterogeneous agents has been proved by Coles (1985).
} 
exhibits the twisted turnpike property (see Mitra (1979), Becker (2005)): the optimal capital accumulation paths starting from different initial capital stocks converge to each other or come together in the limit, but this limit is not itself an optimal stationary program. This is a fundamental property of the heterogeneous agent model and it shows one way in which this model differs significantly from its representative agent counterpart.

The purpose of this paper is to examine whether and under which conditions similar properties hold when the model studied in Le Van and Vailakis (2003) is extended to include an endogenous non-reproducible factor such as labor.

The analysis in Le Van and Vailakis (2003) is carried out by exploiting the so called reduced form model associated with the welfare maximization problem. The presence of heterogeneous discount factors turns out the reduced-form problem to be nonstationary, making the issue of convergence of optimal paths a nontrivial one. Their argument exploits the fact that the indirect utility function $V_{t}$ associated with the reduced form model is $C^{2}$ in the interior of a set describing the feasible activities in period $t$. This allows them to use the differentiable characterization of supermodularity to show that $V_{t}$ is supermodular. The supermodularity of $V_{t}$ then implies that the stationary problem involving the agents with a discount factor equal to the maximum one, has a unique stable steady state $k^{s}$. Exploiting additional properties of optimal paths, they subsequently show that the optimal capital sequence associated with the initial problem involving all agents converges to $k^{s}$.

Several complications arise by applying a similar method of proof in the presence of elastic labor supply. The problems arise largely from the fact that we cannot exclude the existence of corner solutions in the welfare maximization problem $^{2}$. More precisely, we cannot ensure that all consumers supply labor at any period. As a consequence, the indirect utility function $V_{t}$ associated with the reduced form model is not necessarily $C^{2}$ in the interior of the set that describes feasible activities. Hence, one cannot use the differentiable characterization of supermodilarity to prove that $V_{t}$ is supermodular. To overcome the problem and establish the supermodularity of $V_{t}$, we employ an alternative argument based on a iterative procedure in which a sequence of functions, $\left(V_{t}^{n}\right)_{n}$, are shown to be supermodular and to be converging to the function $V_{t}$.

Other issues are associated with the properties of optimal paths. Several proofs in Le Van and Vailakis (2003) do not apply in the presence of elastic labor supply. New and general arguments are given to establish the validity of those properties.

The paper is organised as follows. Section 2 describes the model. In section 3

\footnotetext{
${ }^{2}$ Similar problems arise in incomplete market models (see Becker and Foias (1987) and more recently Datta et al. (2005)).
} 
we present its reduced-form counterpart and establish some preliminary results. Section 4 deals with the dynamic equilibrium properties of optimal paths.

\section{The model}

We consider an intertemporal one-sector model with $m \geq 1$ consumers and one firm. At each period, individuals consume a quantity $c_{i, t}$ and decide how to divide the available time, normalized at 1 , between leisure activities $l_{i, t}$, and work $L_{i, t}$. Preferences are represented by a functional that takes the usual additively separable form:

$$
\sum_{t=0}^{\infty} \beta_{i}^{t} u^{i}\left(c_{i, t}, l_{i, t}\right)
$$

where $u^{i}$ denotes the instantaneous utility function and $\beta_{i} \in(0,1)$ is the discount factor.

The initial endowment of capital, the single reproducible factor in the economy, is denoted by $k_{0} \geq 0$. Technology is described by a gross production function $F$. Capital evolves according to:

$$
k_{t+1}=(1-\delta) k_{t}+I_{t}
$$

where $I_{t}$ is gross investment and $\delta \in(0,1)$ is the rate of depreciation for capital.

At each period, the economy's resource constraints, restricting the allocation of income and time, are:

$$
\sum_{i=1}^{m} c_{i, t}+I_{t} \leq F\left(k_{t}, L_{t}\right)+(1-\delta) k_{t}, \sum_{i=1}^{m} L_{i, t}=L_{t} .
$$

We next specify a set of restrictions imposed on preferences and production technology. The assumptions on period utility function $u^{i}: \mathbb{R}_{+}^{2} \rightarrow \mathbb{R}$ are as follows:

Assumption U1: $u^{i}$ is continuous, strictly concave, increasing on $\mathbb{R}_{+}^{2}$ and strictly increasing on $\mathbb{R}_{++}^{2}$.

Assumption U2: $u^{i}(0,0)=0$.

Assumption U3: $u^{i}$ is twice continuously differentiable on $\mathbb{R}_{++}^{2}$ with partial derivatives satisfying the Inada conditions: $\lim _{c \rightarrow 0} u_{c}^{i}(c, l)=+\infty, \forall l>0$ and $\lim _{l \rightarrow 0} u_{l}^{i}(c, l)=+\infty, \forall c>0$.

Assumption U4: For all $c>0$ and $l>0$, the cross partial derivative $u_{c l}^{i}$ has a constant sign. In addition, we require the first and second partial derivatives to verify the following condition:

$$
\frac{u_{c c}^{i}}{u_{c}^{i}} \leq \frac{u_{c l}^{i}}{u_{l}^{i}}
$$


Remark 1 Assuming that $\frac{u_{c c}}{u_{c}} \leq \frac{u_{c l}}{u_{l}}$ in $\boldsymbol{U}_{4}$ implies that leisure is a normal good. When $u_{c l} \geq 0$, the condition is trivially satisfied. In case where $u_{c l}<0$, it restricts in magnitude. The condition is satisfied for the class of homothetic preferences, $u(c, l)=m[h(c, l)]$, where $h$ is a homogeneous function of degree 1 and $m$ is a monotone increasing transformation. Examples of period utilities with $u_{c l}<0$ that verify this condition include the family of functional forms, $u(c, l)=\frac{1}{1-\sigma}\left[c^{\alpha} U(l)\right]^{1-\sigma}$, where $\sigma>1, \alpha \in(0,1)$ and $U$ is a concave function.

The assumptions on the production function $F: \mathbb{R}_{+}^{2} \rightarrow \mathbb{R}_{+}$are as follows:

Assumption F1: $F$ is continuous, concave, increasing on $\mathbb{R}_{+}^{2}$ and strictly increasing on $\mathbb{R}_{++}^{2}$.

Assumption F2: $F(0,0)=0$.

Assumption F3: $F$ is twice continuously differentiable on $\mathbb{R}_{++}^{2}$ with partial derivatives satisfying the Inada conditions: $\lim _{k \rightarrow 0} F_{k}(k, 1)=+\infty, \lim _{k \rightarrow+\infty}$ $F_{k}(k, m)=0$ and $\lim _{L \rightarrow 0} F_{L}(k, L)=+\infty, \forall k>0$.

Assumption F4: For all $k>0$ and $L>0, F_{k L} \geq 0$ and $F_{k k}<0$.

We conclude this section by introducing some notation.

Let $f\left(k_{t}, L_{t}\right)=F\left(k_{t}, L_{t}\right)+(1-\delta) k_{t}$. Under the previous assumptions we have: $\lim _{k \rightarrow 0} f_{k}(k, 1)=+\infty, \lim _{k \rightarrow+\infty} f_{k}(k, m)<1$ and $\lim _{L \rightarrow 0} f_{L}(k, L)=+\infty$. Consider the set of feasible capital sequences:

$$
\Pi\left(k_{0}\right)=\left\{\mathbf{k} \in\left(\mathbb{R}_{+}\right)^{\infty}: 0 \leq k_{t+1} \leq f\left(k_{t}, m\right), \forall t\right\} .
$$

Let $\mathbf{c}_{i}=\left(c_{i, t}\right)_{t \geq 0}, \mathbf{l}_{i}=\left(l_{i, t}\right)_{t \geq 0}$ and $\mathbf{L}_{i}=\left(L_{i, t}\right)_{t \geq 0}$ denote consumer's $i$ consumption, leisure-work intertemporal allocation. The vector of nonnegative sequences $\left(\mathbf{c}_{i}, \mathbf{l}_{i}, \mathbf{L}_{i}\right)_{i=1}^{m}$ is said to be feasible from $k_{0} \geq 0$, if there exists a sequence $\mathbf{k} \in \Pi\left(k_{0}\right)$, such that for any $t,\left(\left(c_{i, t}, l_{i, t}, L_{i, t}\right)_{i=1}^{m}, k_{t}\right)$ satisfies the economy's resource constraints together with the individual constraints $l_{i, t}+L_{i, t} \leq 1$. The set of feasible from $k_{0}$ consumption, leisure-labor allocations is denoted by $\Sigma\left(k_{0}\right)$.

\section{Planner's Problem}

The planner's welfare function is taken to be a weighted function of the underlying households' intertemporal functions. Let $\Delta=\left\{\lambda \in \mathbb{R}_{+}^{m} \mid \sum_{i=1}^{m} \lambda_{i}=1\right\}$. Given a vector of welfare weights $\lambda \in \Delta$ the social planner maximizes: 


$$
\begin{array}{ll}
\max & \sum_{i=1}^{m} \lambda_{i} \sum_{t=0}^{\infty} \beta_{i}^{t} u^{i}\left(c_{i, t}, l_{i, t}\right) \\
\text { s.t. } & \sum_{i=1}^{m} c_{i, t}+k_{t+1} \leq f\left(k_{t}, \sum_{i=1}^{m} L_{i, t}\right), \forall t \\
& c_{i, t} \geq 0, l_{i, t} \geq 0, L_{i, t} \geq 0, l_{i, t}+L_{i, t} \leq 1, \forall i, \forall t \\
& k_{t} \geq 0, \forall t \text { and } k_{0} \text { given. }
\end{array}
$$

It is well known that any Pareto-efficient allocation can be represented as the solution to problem $(P)$. In other words, by varying the welfare weights it is possible to trace the economy's utility possibility frontier. This procedure can also be used to prove the existence of a price system that supports Paretooptima and characterize competitive equilibria as a set of welfare weights such that the associated transfer payments are zero (Negishi approach).

\subsection{Preliminary results}

Since $u^{i}$ and $F$ are assumed to be strictly increasing, $\left(\mathbf{L}_{i}\right)_{i=1}^{m}$ can be dropped from the list of planner's choices. Consider the technology set $D$ :

$$
D:=\left\{(k, y) \in \mathbb{R}_{+}^{2}: 0 \leq y \leq f(k, m)\right\}
$$

and define the correspondence $\Gamma$ :

$$
(k, y) \in D \rightarrow\left\{\left(c_{i}, l_{i}\right)_{i=1}^{m}: \sum_{i=1}^{m} c_{i}+y \leq f\left(k, \sum_{i=1}^{m}\left(1-l_{i}\right)\right), c_{i} \geq 0, l_{i} \in[0,1], \forall i\right\} .
$$

Given $\lambda \in \Delta$, let $I=\left\{i \mid \lambda_{i}>0\right\}, \beta=\max \left\{\beta_{i} \mid i \in I\right\}, I_{1}=\left\{i \in I \mid \beta_{i}=\beta\right\}$ and $I_{2}=\left\{i \in I \mid \beta_{i}<\beta\right\}$.

Remark 2 Let $\left(\left(\mathbf{c}_{i}^{*}, \mathbf{l}_{i}^{*}\right)_{i}, \mathbf{k}^{*}\right)$ denote a solution to problem $(P)$. It is easy to show that if $\lambda_{i}=0$ for some $i$, then $c_{i, t}^{*}=0$ and $l_{i, t}^{*}=0$ for any $t \geq 0$.

Denote $\zeta:=\left(\zeta_{i}\right)_{i \in I_{2}} \in[0,1]^{\# I_{2}}$. Consider the function $V: \Delta \times[0,1]^{\# I_{2}} \times D \rightarrow$ $\mathbb{R}_{+}$:

$$
\begin{gathered}
V(\lambda, \zeta, k, y)=\max \left[\sum_{i \in I_{1}} \lambda_{i} u^{i}\left(c_{i}, l_{i}\right)+\sum_{i \in I_{2}} \lambda_{i} \zeta_{i} u^{i}\left(c_{i}, l_{i}\right)\right] \\
\text { s.t. } \sum_{i \in I} c_{i}+y \leq f\left(k, m-\sum_{i \in I} l_{i}\right) \\
c_{i} \geq 0, l_{i} \in[0,1], \forall i \in I .
\end{gathered}
$$


Let

$\left(c_{i}(\lambda, \zeta, k, y), l_{i}(\lambda, \zeta, k, y)\right)_{i \in I}=\arg \max \left\{\sum_{i \in I} \lambda_{i} \zeta_{i} u^{i}\left(c_{i}, l_{i}\right):\left(c_{i}, l_{i}\right)_{i \in I} \in \Gamma(k, y)\right\}$.

For any $t \geq 0$, for any $\zeta \in[0,1]^{\# I_{2}}$, we use the notation $\zeta^{t}=\left(\zeta_{i}^{t}\right)_{i \in I_{2}}$. Fix $\zeta=\left(\frac{\beta_{i}}{\beta}\right)_{i \in I_{2}}$. In this case, for any $t$, we denote:

$$
\begin{aligned}
V_{t}(\lambda, \zeta, k, y):= & V\left(\lambda, \zeta^{t}, k, y\right) \\
= & \max \left[\sum_{i \in I_{1}} \lambda_{i} u^{i}\left(c_{i}, l_{i}\right)+\sum_{i \in I_{2}} \lambda_{i}\left(\frac{\beta_{i}}{\beta}\right)^{t} u^{i}\left(c_{i}, l_{i}\right)\right] \\
& \text { s.t. } \sum_{i \in I} c_{i}+y \leq f\left(k, m-\sum_{i \in I} l_{i}\right) \\
& c_{i} \geq 0, l_{i} \in[0,1], \forall i \in I .
\end{aligned}
$$

Consider subsequently the following intertemporal problem:

$$
\begin{array}{ll}
\max & \sum_{t=0}^{\infty} \beta^{t} V_{t}\left(\lambda, \zeta, k_{t}, k_{t+1}\right) \\
\text { s.t. } & 0 \leq k_{t+1} \leq f\left(k_{t}, m\right), \forall t \\
& k_{0} \geq 0 \text { is given. }
\end{array}
$$

The following proposition shows that problems $(P)$ and $(Q)$ are equivalent. More precisely we have:

Proposition 1 Let $k_{0} \geq 0$ be given. Under assumptions U1, F1:

i) If $\left(\left(\mathbf{c}_{i}^{*}, \mathbf{l}_{i}^{*}\right)_{i}, \mathbf{k}^{*}\right)$ is a solution to problem $(P)$, then $\mathbf{k}^{*}$ is a solution to problem $(Q)$.

ii) If $\mathbf{k}^{*}$ is a solution to problem $(Q)$, then there exists $\left(\mathbf{c}_{i}^{*}, \mathbf{l}_{i}^{*}\right)_{i}$ such that $\left(\left(\mathbf{c}_{i}^{*}, \mathbf{l}_{i}^{*}\right)_{i}, \mathbf{k}^{*}\right)$ is a solution to problem $(P)$.

Proof: It is easy.

The next step is to study the properties of function $V_{t}$. Working in this direction we need the following result.

Lemma 1 Under Assumptions U1-U2, F1-F2:

i) If $F(0, L)>0$, then $\Gamma$ is continuous at any $(k, y) \in D$

ii) If $F(0, L)=0$, then $\Gamma$ is upper hemicontinuous at $(0,0)$ and continuous at any $(k, y) \in D$ with $k>0$. 
Proof: i) Let $\left(k^{n}, y^{n}\right) \in D$ be a sequence that converges to some $(k, y) \in D$. Let also $\left(c_{i}^{n}, l_{i}^{n}\right)_{i}$ be a sequence such that $\left(c_{i}^{n}, l_{i}^{n}\right)_{i} \in \Gamma\left(k^{n}, y^{n}\right), \forall n \in \mathbb{N}$. Since $l_{i}^{n} \in[0,1], c_{i}^{n} \leq f\left(k^{n}, m\right)$ and $k^{n} \rightarrow k$, there exists a subsequence $\left(c_{i}^{n}, l_{i}^{n}\right)_{i}$ that converges to some $\left(c_{i}, l_{i}\right)_{i}$. Since $\sum_{i=1}^{m} c_{i}^{n}+y^{n} \leq f\left(k^{n}, \sum_{i=1}^{m}\left(1-l_{i}^{n}\right)\right)$, it follows that $\left(c_{i}, l_{i}\right)_{i} \in \Gamma(k, y)$.

We next show that $\Gamma$ is lower hemicontinuous at any $(k, y) \in D$.

Let $\left(k^{n}, y^{n}\right) \in D$ be a sequence that converges to some $(k, y) \in D$. Take $\left(c_{i}, l_{i}\right)_{i} \in$ $\Gamma(k, y)$. We will show that there exists $N \geq 1$ and a sequence $\left(c_{i}^{n}, l_{i}^{n}\right)_{i}$ such that $\left(c_{i}^{n}, l_{i}^{n}\right)_{i} \in \Gamma\left(k^{n}, y^{n}\right), \forall n \geq N$ and $\left(c_{i}^{n}, l_{i}^{n}\right)_{i} \rightarrow\left(c_{i}, l_{i}\right)_{i}$. We consider three cases:

Case 1: $\sum_{i=1}^{m} c_{i}+y<f\left(k, \sum_{i=1}^{m}\left(1-l_{i}\right)\right)$.

Observe that for $n$ large enough, $\sum_{i=1}^{m} c_{i}+y^{n}<f\left(k^{n}, \sum_{i=1}^{m}\left(1-l_{i}\right)\right)$. In this case, let $c_{i}^{n}=c_{i}$ and $l_{i}^{n}=l_{i}$.

Case 2: $\sum_{i=1}^{m} c_{i}+y=f\left(k, \sum_{i=1}^{m}\left(1-l_{i}\right)\right)$ and $\sum_{i=1}^{m} c_{i}>0$.

Denote by $I^{\prime}$ the set of agents such that $c_{i}>0$. In this case there exists $N$ such that $y^{n}<f\left(k^{n}, \sum_{i=1}^{m}\left(1-l_{i}\right)\right), \forall n \geq N$. Let $\xi=f\left(k, \sum_{i=1}^{m}\left(1-l_{i}\right)\right)-y$ and $\xi^{n}=f\left(k^{n}, \sum_{i=1}^{m}\left(1-l_{i}\right)\right)-y^{n}, \forall n \geq N$. Since $\xi^{n} \rightarrow \xi$, for $N$ large enough, it folows that $c_{i}+\frac{\xi^{n}-\xi}{\# I^{\prime}}>0, \forall n \geq N$. For any $n \geq N$, let $c_{i}^{n}=c_{i}+\frac{\xi^{n}-\xi}{\# I^{\prime}}$ if $i \in I^{\prime}$, $c_{i}^{n}=0$ if $i \notin I^{\prime}$ and $l_{i}^{n}=l_{i}$ for any $i$.

Case 3: $\sum_{i=1}^{m} c_{i}+y=f\left(k, \sum_{i=1}^{m}\left(1-l_{i}\right)\right)$ and $c_{i}=0, \forall i$.

We have $y=f\left(k, \sum_{i=1}^{m}\left(1-l_{i}\right)\right)$. We consider three subcases.

a) Assume that $l_{i}=0, \forall i$.

In this case, $y=f(k, m)$. Let $c_{j}^{n}=f\left(k^{n}, m\right)-y^{n}$ for some $j, c_{i}^{n}=0, \forall i \neq j$ and $l_{i}^{n}=0, \forall i$.

b) Assume that there exists a subset of agents, denoted $I^{\prime}$, such that $l_{i} \in$ $(0,1)$ for any $i \in I^{\prime}$.

In this case, $y=f\left(k, \sum_{i=1}^{m}\left(1-l_{i}\right)\right)<f(k, m)$. Assume first that there exists a subsequence $\left(k^{n}, y^{n}\right)$ such that $y^{n} \leq f\left(k^{n}, \sum_{i=1}^{m}\left(1-l_{i}\right)\right)$. In this case, let $c_{j}^{n}=$ $f\left(k^{n}, \sum_{i=1}^{m}\left(1-l_{i}\right)\right)-y^{n}$ for some $j, c_{i}^{n}=0$ for $i \neq j$ and $l_{i}^{n}=l_{i}, \forall i$. Assume next that there exists a subsequence $\left(k^{n}, y^{n}\right)$ such that $y^{n}>f\left(k^{n}, \sum_{i=1}^{m}\left(1-l_{i}\right)\right)$. Choose some agent $j \in I^{\prime}$. Define the decreasing functions $\psi_{j}:[0,1] \rightarrow \mathbb{R}$ and $\psi_{j}^{n}:[0,1] \rightarrow \mathbb{R}$ for $n \in \mathbb{N}$ as follows:

$$
\begin{aligned}
& \psi_{j}(\xi)=f\left(k,(1-\xi)+m-1-\sum_{i \neq j} l_{i}\right)-y \\
& \psi_{j}^{n}(\xi)=f\left(k^{n},(1-\xi)+m-1-\sum_{i \neq j} l_{i}\right)-y^{n} .
\end{aligned}
$$

Note that $\psi_{j}\left(l_{j}\right)=0$. Observe that there exists $N$ large enough such that $\psi^{n}(0)>0, \forall n \geq N$. Moreover, $\psi_{j}^{n}\left(l_{j}\right)=f\left(k^{n}, \sum_{i=1}^{m}\left(1-l_{i}\right)\right)-y^{n}<0, \forall n \geq N$. By the Intermediate Value Theorem, there exists $\xi^{n} \in\left(0, l_{j}\right)$ such that $\psi_{j}^{n}\left(\xi^{n}\right)=$ 0 . For any $n \geq N$, let $l_{j}^{n}=\xi^{n}, l_{i}^{n}=l_{i}, \forall i \neq j$ and $c_{i}^{n}=0, \forall i$.

c) Assume that $l_{i}=1, \forall i$. 
In this case, $y=f(k, 0)$. Assume first that there exits a subsequence $\left(k^{n}, y^{n}\right)$ such that $y^{n} \leq f\left(k^{n}, 0\right)$. In this case, let $c_{j}^{n}=f\left(k^{n}, 0\right)-y^{n}$ for some $j, c_{i}^{n}=0$, $\forall i \neq j$ and $l_{i}^{n}=1, \forall i$. Assume next that there exists a subsequence $\left(k^{n}, y^{n}\right)$ such that $y^{n}>f\left(k^{n}, 0\right)$. Fix some agent $j \in\{1, \ldots m\}$. Define the decreasing functions $\psi_{j}:[0,1] \rightarrow \mathbb{R}$ and $\psi_{j}^{n}:[0,1] \rightarrow \mathbb{R}$ for $n \in \mathbb{N}:$

$$
\begin{aligned}
\psi_{j}(\xi) & =f(k,(1-\xi))-y \\
\psi_{j}^{n}(\xi) & =f\left(k^{n},(1-\xi)\right)-y^{n} .
\end{aligned}
$$

Note that $\psi_{j}(1)=0$. Observe that there exists $N$ large enough such that $\psi_{j}^{n}(0)>0, \forall n \geq N$. Moreover, $\psi_{j}^{n}(1)=f\left(k^{n}, 0\right)-y^{n}<0, \forall n \geq N$. By the Intermediate Value Theorem, there exists $\xi^{n} \in(0,1)$ such that $\psi_{j}^{n}\left(\xi^{n}\right)=0$. For any $n \geq N$, let $l_{j}^{n}=\xi^{n}, l_{i}^{n}=1, \forall i \neq j$ and $c_{i}^{n}=0, \forall i$.

ii) The continuity of $\Gamma$ at any $(k, y) \in D$ with $k>0$ follows in a similar way as in claim (i). To see why the lower hemicontinuity of $\Gamma$ fails at $(0,0)$ when $F(0, L)=0$, observe that in this case $D=\{(0,0)\}$ and

$$
\Gamma(0,0)=\left\{\left(c_{i}, l_{i}\right)_{i}: c_{i}=0 \text { and } l_{i} \in[0,1], \forall i\right\} .
$$

Choose $\left(c_{i}, l_{i}\right)_{i} \in \Gamma(0,0)$ such that $c_{i}=0$ and $l_{i}>0$ for some $i$. Consider next a sequence $\left(k^{n}, y^{n}\right)$ such that $y^{n}=f\left(k^{n}, m\right)$ and $\left(k^{n}, y^{n}\right) \rightarrow(0,0)$. Note that there is no sequence $\left(c_{i}^{n}, l_{i}^{n}\right)_{i}$ such that $\left(c_{i}^{n}, l_{i}^{n}\right)_{i} \in \Gamma\left(k^{n}, y^{n}\right)$ and $\left(c_{i}^{n}, l_{i}^{n}\right)_{i} \rightarrow\left(c_{i}, l_{i}\right)_{i}$.

Proposition 2 Assume $\boldsymbol{U 1 - U} 3$ and $\boldsymbol{F 1 - F 3}$. Then,

i) The function $V: \Delta \times[0,1]^{\# I_{2}} \times D \rightarrow \mathbb{R}_{+}$is increasing in $k$, decreasing in $y$ and strictly concave in $(k, y)$.

ii) The function $V$ is upper semicontinuous on $[0,1]^{\# I_{2}} \times D$ and continuous at any $(\zeta, k, y) \in[0,1]^{\# I_{2}} \times D$ with $k>0$.

iii) The functions $c_{i}: \Delta \times[0,1]^{\# I_{2}} \times D \rightarrow \mathbb{R}_{+}$and $l_{i}: \Delta \times[0,1]^{\# I_{2}} \times D \rightarrow[0,1]$ are continuous at any $(\zeta, k, y) \in[0,1]^{\# I_{2}} \times$ int D.

iv) Let $(k, y) \in D$ be such that $0 \leq y<f(k, m)$. If $\left(c_{i}^{*}, l_{i}^{*}\right)_{i \in I}$ is a solution to the static maximization problem $(S)$, then, $c_{i}^{*}>0$ and $l_{i}^{*}>0$ for any $i \in I$.

v) $V_{t}$ is differentiable at any $(k, y) \in$ int $D$ with partial derivatives given by:

$$
\begin{aligned}
& \frac{\partial V_{t}(\lambda, \zeta, k, y)}{\partial k}=\mu_{t} f_{k}\left(k, m-\sum_{i \in I} l_{i}^{*}\right) \\
& \frac{\partial V_{t}(\lambda, \zeta, k, y)}{\partial y}=-\mu_{t}
\end{aligned}
$$

where $\mu_{t}=\lambda_{i}\left(\frac{\beta_{i}}{\beta}\right)^{t} u_{c}^{i}\left(c_{i}^{*}, l_{i}^{*}\right), \forall i \in I$.

Proof: The proof of (i) follows from standard arguments. Claims (ii) and (iii) follow from the Maximum Theorem. 
(iv) Since $0 \leq y<f(k, m)$, there exists $\varepsilon>0$ such that $0<y+\varepsilon<$ $f(k, m-\varepsilon)$. Letting $c_{i}=\frac{\varepsilon}{\# I}$ and $l_{i}=\frac{\varepsilon}{\# I}, \forall i \in I$, the Slater condition is satisfied. Hence, there exist Lagrange multipliers $\mu_{t}\left(\lambda, \zeta^{t}, k, y\right) \in \mathbb{R}_{+}$associated with the constraint $\sum_{i} c_{i}+y \leq f\left(k, m-\sum_{i \in I} l_{i}\right)$ and $\eta_{i, t}\left(\lambda, \zeta^{t}, k, y\right) \in \mathbb{R}_{+}$ associated with the constraints $l_{i} \leq 1$ such that $\left(\left(c_{i}^{*}\right)_{i \in I},\left(l_{i}^{*}\right)_{i \in I}, \mu_{t},\left(\eta_{i, t}\right)_{i \in I}\right)$ maximizes the associated Lagrangian :

$\mathcal{L}=\sum_{i \in I} \lambda_{i}\left(\frac{\beta_{i}}{\beta}\right)^{t} u^{i}\left(c_{i}, l_{i}\right)-\mu_{t}\left[\sum_{i \in I} c_{i}+y-f\left(k, m-\sum_{i \in I} l_{i}\right)\right]-\sum_{i \in I} \eta_{i, t}\left(l_{i}-1\right)$.

The fact that $c_{i}^{*}>0$ and $l_{i}^{*}>0, \forall i \in I$ is a consequence of the Inada conditions imposed on period utilities.

Claim (v) follows from a direct application of Corollary 7.3.1 in Florenzano, Le Van and Gourdel (2001).

Since $f_{k}(\infty, m)<1$, there exists some $\bar{k}$ such that $f(\bar{k}, m)=\bar{k}$. It is easy to show that $\mathbf{k} \in \Pi\left(k_{0}\right)$ implies that $k_{t} \leq A\left(k_{0}\right)=\max \left\{k_{0}, \bar{k}\right\}$ for all $t$. This in turn implies that $\Pi\left(k_{0}\right)$ is included in a compact set for the product topology. Since $f$ is continuous, the set $\Pi\left(k_{0}\right)$ is closed for the product topology, and therefore is compact in this topology. Define the function $U: \mathbb{R}_{+} \times \Pi\left(k_{0}\right) \rightarrow \mathbb{R}_{+}$:

$$
U\left(k_{0}, \mathbf{k}\right)=\sum_{t=0}^{\infty} \beta^{t} V_{t}\left(\lambda, k_{t}, k_{t+1}\right) .
$$

We have the following result.

Lemma 2 i) The correspondence $\Pi$ is continuous for the product topology. ii) $U\left(k_{0}, \cdot\right)$ is upper semicontinuous on $\Pi\left(k_{0}\right)$ for the product topology.

Proof: Refer to Le Van and Morhaim (2002, Lemma 2, Proposition 2).

It follows that problem $(Q)$ is equivalent to the maximization of an upper semicontinuous function over a compact set, and therefore it admits a solution. Observe also that the strict concavity of $V_{t}$ implies that the solution is unique.

Proposition 3 For all $k_{0} \geq 0$, there is a unique optimal accumulation path.

\subsection{Value function-Bellman equation-Optimal policy}

One way to make any further analysis easier is to work with the value function. Let $\zeta=\left(\zeta_{i}\right)_{i \in I_{2}} \in[0,1]^{\# I_{2}}$. Given $T \geq 0$, define the function $W_{T}:\left(\zeta, k_{0}\right) \in$ 


$$
[0,1]^{\# I_{2}} \times \mathbb{R}_{+} \rightarrow \mathbb{R}_{+}:
$$

$$
\begin{aligned}
W_{T}\left(\zeta, k_{0}\right):= & W\left(\zeta^{T}, k_{0}\right) \\
= & \max \sum_{t=0}^{\infty} \beta^{t} V_{t}\left(\lambda, \zeta^{T}, k_{t}, k_{t+1}\right) \\
& \text { s.t. } 0 \leq k_{t+1} \leq f\left(k_{t}, m\right), \forall t \\
\quad & k_{0} \geq 0 \text { is given. }
\end{aligned}
$$

It is obvious that when $\zeta=\left(\frac{\beta_{i}}{\beta}\right)_{i \in I_{2}}, W_{0}\left(\zeta, k_{0}\right)$ is the value function associated with problem $(Q)$. In infinite-horizon problems with time-invariant period return functions (stationary problems) the value function is a function of the initial state alone. In the above problem the period return function is timedependent, so the problem is a nonstationary one. In this case, as the time index on $W$ indicates, the value function is also time dependent.

Proposition 4 The function $W_{T}:(\zeta, k) \in[0,1]^{\# I_{2}} \times \mathbb{R}_{+} \rightarrow \mathbb{R}_{+}$is strictly increasing and strictly concave in $k$, nonnegative with $W(\zeta, k)>0$ for $k>0$, and continuous at any $(\zeta, k) \in[0,1]^{\# I_{2}} \times \mathbb{R}_{+}$.

Proof: It follows from standard arguments.

The next proposition states formally what is known as the Principle of Optimality. It will help us characterize basic properties of optimal paths.

Proposition 5 The value function solves the Bellman equation, i.e

$$
\forall t, \forall k, W_{t}(\zeta, k)=\max \left\{V_{t}(\lambda, \zeta, k, y)+\beta W_{t+1}(\zeta, y): 0 \leq y \leq f(k, m)\right\}
$$

and for all $k_{0} \geq 0$, a feasible path $\mathbf{k}$ is optimal, if and only if,

$$
W_{t}\left(\zeta, k_{t}\right)=V_{t}\left(\lambda, \zeta, k_{t}, k_{t+1}\right)+\beta W_{t+1}\left(\zeta, k_{t+1}\right)
$$

holds for all $t$.

We next define the function $\varphi_{T}:(\zeta, k) \in[0,1]^{\# I_{2}} \times \mathbb{R}_{+} \rightarrow \mathbb{R}_{+}:$

$\varphi_{T}(\zeta, k):=\varphi\left(\zeta^{T}, k\right)=\arg \max \left\{V\left(\lambda, \zeta^{T}, k, y\right)+\beta W\left(\zeta^{T+1}, y\right): 0 \leq y \leq f(k, m)\right\}$.

When $\zeta=\left(\frac{\beta_{i}}{\beta}\right)_{i \in I_{2}}$, it follows that for any $T \geq 0$, for any $k_{T}$ :

$$
\begin{aligned}
k_{T+t}^{*} & =\varphi\left(\zeta^{T+t-1}, k_{T+t-1}^{*}\right) \\
& =\varphi\left(\zeta^{T+t-1}, \varphi\left(\zeta^{T+t-2}, k_{T+t-2}^{*}\right)\right) \\
& =\varphi\left(\zeta^{T+t-1}, \varphi\left(\zeta^{T+t-2}, \ldots, \varphi\left(\zeta^{T}, k_{T}\right) \ldots\right)\right)
\end{aligned}
$$

for $t \geq 1$. For simplicity we write $k_{T+t}^{*}=\varphi^{t}\left(\zeta^{T}, k_{T}\right)$. 
Proposition 6 The function $\varphi$ is continuous at any $(\zeta, k) \in[0,1]^{\# I_{2}} \times \mathbb{R}_{+}$, and therefore uniformly continuous at any $(\zeta, k) \in[0,1]^{\# I_{2}} \times\left[0, A\left(k_{0}\right)\right]$.

Proof: Assume first that $F$ satisfies $F(0, L)>0$. The result follows from the Maximum Theorem. Assume next that $F$ satisfies $F(0, L)=0$. If $k>0$, the continuity of $\varphi$ follows from the Maximum Theorem. When $k=0$, feasibility implies that $\varphi(\zeta, k)=0$. Let $\left(\zeta_{n}, k_{n}\right)_{n} \in[0,1]^{\# I_{2}} \times \mathbb{R}_{+}$be a sequence such that $0 \leq \varphi\left(\zeta_{n}, k_{n}\right) \leq f\left(k_{n}, m\right)$ and $k_{n} \rightarrow 0$. Since $f(0, m)=0$, it follows that the sequence of maximizers converges to 0 as $n \rightarrow+\infty$.

Consider next the planner's problem involving only the agents having a discount factor equal to the maximum one. Let $\underset{\sim}{0}:=(0)_{i \in I_{2}}$ denote the zero vector in $\mathbb{R}^{\# I_{2}}$. Define the time invariant function $\widehat{V}: \Delta \times D \rightarrow \mathbb{R}_{+}$:

$$
\begin{aligned}
\widehat{V}(\lambda, k, y):= & V(\lambda \underset{\sim}{0}, k, y) \\
= & \max \sum_{i \in I_{1}} \lambda_{i} u^{i}\left(c_{i}, l_{i}\right) \\
& \text { s.t. } \sum_{i \in I_{1}} c_{i}+y \leq f\left(k, m-\sum_{i \in I_{1}} l_{i}\right) \\
& \quad c_{i} \geq 0, l_{i} \in[0,1], \forall i \in I_{1} .
\end{aligned}
$$

The intertemporal problem associated with the indirect utility $\widehat{V}$ is now stationary:

$$
\begin{aligned}
\widehat{W}\left(k_{0}\right):= & \left.W \underset{\sim}{0}, k_{0}\right) \\
= & \max \sum_{t=0}^{\infty} \beta^{t} \widehat{V}\left(\lambda, k_{t}, k_{t+1}\right) \\
& \text { s.t. } 0 \leq k_{t+1} \leq f\left(k_{t}, m\right), \forall t \\
& k_{0} \geq 0, \text { is given. }
\end{aligned}
$$

The optimal policy function for this problem is given by:

$$
\varphi(\underset{\sim}{0}, k)=\arg \max \{\widehat{V}(\lambda, k, y)+\beta \widehat{W}(y): 0 \leq y \leq f(k, m)\} .
$$

If $\mathbf{k}^{*}$ is the optimal solution to problem $(\widehat{Q})$, then obviously $k_{t}^{*}=\varphi\left(\underset{\sim}{0}, k_{t-1}^{*}\right)=$ $\varphi^{t}\left(\underset{\sim}{0}, k_{0}\right)$.

Let $k_{0} \geq 0$. It is easy to check that for any feasible allocation $\left(\left(\mathbf{c}_{i}, \mathbf{l}_{i}\right)_{i}, \mathbf{k}\right)$ from $k_{0}$, we have:

$$
u^{i}\left(c_{i, t}, l_{i, t}\right) \leq u^{i}\left(f\left(A\left(k_{0}\right), m\right), 1\right)=B\left(k_{0}\right), \forall i
$$

This in turn implies that for any $t$, for any $(k, y) \in D$ : 


$$
\widehat{V}(\lambda, k, y) \leq V_{t}(\lambda, \zeta, k, y) \leq\left(\frac{\max _{i \in I_{2}} \beta_{i}}{\beta}\right)^{t} C\left(k_{0}\right)+\widehat{V}(\lambda, k, y)
$$

where $C\left(k_{0}\right)=\sum_{i \in I_{2}} B\left(k_{0}\right)$. Let $\varepsilon>0$. Since $\left(\frac{\max _{i \in I_{2}} \beta_{i}}{\beta}\right)<1$, there exists $T$ independent of $k_{0}$ such that for any $t$ :

$$
\widehat{V}(\lambda, k, y) \leq V_{T+t}(\lambda, \zeta, k, y) \leq \varepsilon C\left(k_{0}\right)+\widehat{V}(\lambda, k, y) .
$$

It follows that for any $k_{0} \geq 0$, for any $T^{\prime} \geq T$ :

$$
\widehat{W}\left(k_{0}\right) \leq W_{T^{\prime}}\left(\zeta, k_{0}\right) \leq \varepsilon C\left(k_{0}\right) \frac{1}{1-\beta}+\widehat{W}\left(k_{0}\right) .
$$

Observe that for any sequence $\left(k_{t}\right)_{t=0}^{\infty}$ feasible from $k_{0}$, we have $C\left(k_{t}\right) \leq C\left(k_{0}\right)$. Hence, the following inequalities hold:

$$
\begin{gathered}
\widehat{V}\left(\lambda, k_{t}, k_{t+1}\right) \leq V_{t}\left(\lambda, \zeta, k_{t}, k_{t+1}\right) \leq \varepsilon C\left(k_{0}\right)+\widehat{V}\left(\lambda, k_{t}, k_{t+1}\right), \forall t \geq T, \\
\widehat{W}\left(k_{t}\right) \leq W_{t}\left(\zeta, k_{t}\right) \leq \varepsilon C\left(k_{0}\right) \frac{1}{1-\beta}+\widehat{W}\left(k_{t}\right), \forall t \geq T .
\end{gathered}
$$

Consider a feasible capital sequence $\left(k_{t}\right)_{t=0}^{\infty}$ starting from some $k_{0} \geq 0$. Using the previous results, for any subsequence $\left(t_{n}\right)_{n=1}^{\infty}$ such that $k_{t_{n}} \rightarrow k \geq 0$ and $k_{t_{n}+1} \rightarrow k^{\prime} \geq 0$, we have:

$$
\lim _{n \rightarrow \infty} V_{t_{n}}\left(\lambda, \zeta, k_{t_{n}}, k_{t_{n}+1}\right)=\widehat{V}\left(\lambda, k, k^{\prime}\right) \text { and } \lim _{n \rightarrow \infty} W_{t_{n}}\left(\zeta, k_{t_{n}}\right)=\widehat{W}(k)
$$

\section{Dynamic Equilibrium Properties}

It is mentioned in the introduction that when labor is elastic one cannot exclude the existence of corner solutions in the welfare maximization problem. The presence of corner solution is related to the possibility of having consumers not supplying labor in some periods. It is a direct consequence that in this case the indirect utility function $V_{t}$ is not necessarily $C^{2}$ in the interior of $D$. As a result one cannot use the differentiable characterization of supermodularity to prove that the indirect utility function $V_{t}$ is supermodular. To prove the validity of this property in our setting we employ an alternative argument based on a iterative procedure in which a sequence of functions are shown to be supermodular and to be converging to the function $V_{t}$. In what follows we deal with the construction of such a sequence. 
Let $\alpha \in(0,1)$ and $\nu \in(0,1]$. Consider the production function $\widetilde{f}$ defined as follows:

$$
\widetilde{f}\left(\nu, k,\left(1-l_{i}\right)_{i}\right):=f\left(k, m-\sum_{i=1}^{m} l_{i}\right)+\nu \sum_{i=1}^{m}\left(1-l_{i}\right)^{\alpha}
$$

Observe that $\lim _{l_{i} \rightarrow 1} \widetilde{f}_{L_{i}}\left(\nu, k,\left(1-l_{i}\right)_{i}\right)=+\infty$. Denote $\tilde{f}(\nu, k, m):=f(k, m)+$ $\nu m$. Let $\widetilde{D}$ be the technology set:

$$
\widetilde{D}:=\left\{(k, y) \in \mathbb{R}_{+}^{2}: 0 \leq y \leq \widetilde{f}(\nu, k, m)\right\}
$$

and define the correspondence $\widetilde{\Gamma}$ :

$$
\begin{gathered}
(\nu, k, y) \in(0,1] \times \widetilde{D} \rightarrow\left\{\left(c_{i}, l_{i}\right)_{i}: \sum_{i=1}^{m} c_{i}+y \leq \widetilde{f}\left(\nu, k,\left(1-l_{i}\right)_{i}\right), c_{i} \geq 0\right. \\
\left.l_{i} \in[0,1], \forall i\right\}
\end{gathered}
$$

Lemma 3 Under Assumptions U1-U2, F1-F2, $\widetilde{\Gamma}$ continuous at any $(\nu, k, y) \in$ $(0,1] \times \widetilde{D}$

Proof: Observe that $\tilde{f}\left(\nu, 0,\left(1-l_{i}\right)_{i}\right)>0$ for any $\nu \in(0,1]$. The proof is similar to the one used to prove Lemma 1(i).

Given any $\nu \in(0,1]$ denote by $(P(\nu))$ the Pareto optimal problem (analogous to problem $(P))$ associated with an economy with production possibilities described by the function $\tilde{f}$. Let $\left(\left(\mathbf{c}_{i}^{*}(\nu), \mathbf{l}_{i}^{*}(\nu)\right)_{i}, \mathbf{k}^{*}(\nu)\right)$ denote a solution to problem $(P(\nu))$. It is easy to show that $\lambda_{i}=0$ for some $i$ implies $c_{i, t}^{*}(\nu)=0$ and $l_{i, t}^{*}(\nu)=0$ for any $t$. Denote:

$$
\tilde{f}\left(\nu, k,\left(1-l_{i}\right)_{i \in I}\right):=f\left(k, m-\sum_{i \in I} l_{i}\right)+\nu \sum_{i \in I}\left(1-l_{i}\right)^{\alpha}+\nu(m-\# I)
$$

Let $\zeta=\left(\frac{\beta_{i}}{\beta}\right)_{i \in I_{2}}$. In this case,

$$
\begin{aligned}
\widetilde{V}_{t}(\nu, \lambda, \zeta, k, y):= & \widetilde{V}\left(\nu, \lambda, \zeta^{t}, k, y\right) \\
= & \max \left[\sum_{i \in I_{1}} \lambda_{i} u^{i}\left(c_{i}, l_{i}\right)+\sum_{i \in I_{2}} \lambda_{i}\left(\frac{\beta_{i}}{\beta}\right)^{t} u^{i}\left(c_{i}, l_{i}\right)\right](S(\nu)) \\
& \text { s.t. } \sum_{i \in I} c_{i}+y \leq \tilde{f}\left(\nu, k,\left(1-l_{i}\right)_{i \in I}\right) \\
& c_{i} \geq 0, l_{i} \in[0,1], \forall i \in I .
\end{aligned}
$$

is the indirect utility function associated with Problem $(P(\nu))$. In a similar way, 
for any $T \geq 0$, let :

$$
\begin{aligned}
\widetilde{W}_{T}\left(\nu, \zeta, k_{0}\right):= & \widetilde{W}\left(\nu, \zeta^{T}, k_{0}\right) \\
= & \max \sum_{t=0}^{\infty} \beta^{t} \widetilde{V}\left(\nu, \lambda, \zeta^{T+t}, k_{t}, k_{t+1}\right) \quad(Q(\nu)) \\
& \text { s.t. } 0 \leq k_{t+1} \leq \widetilde{f}\left(\nu, k_{t}, m\right), \forall t \\
& k_{0} \geq 0 \text { is given. }
\end{aligned}
$$

be the value function associated with the indirect utility $\widetilde{V}_{t}$. Finally, let

$$
\begin{aligned}
\widetilde{\varphi}_{T}(\nu, \zeta, k) & :=\widetilde{\varphi}\left(\nu, \zeta^{T}, k\right) \\
& =\arg \max \left\{\widetilde{V}\left(\nu, \lambda, \zeta^{T}, k, y\right)+\beta \widetilde{W}\left(\zeta^{T+1}, y\right): 0 \leq y \leq \widetilde{f}(\nu, k, m)\right\}
\end{aligned}
$$

denote the policy function associated with problem $Q(\nu)$. We have the following results.

Proposition 7 Assume $\boldsymbol{U 1 - U} 4$ and $\boldsymbol{F} 1-\boldsymbol{F} 4$. Then,

i) The function $\widetilde{V}_{t}$ is strictly concave in $(k, y)$ and continuous at any $(\nu, k, y) \in$ $(0,1] \times \widetilde{D}$. Moreover,

$$
\lim _{\nu \rightarrow 0} \widetilde{V}_{t}(\nu, \lambda, \zeta, k, y)=V_{t}(\lambda, \zeta, k, y) .
$$

ii) Let $\nu \in(0,1]$ and $(k, y) \in \widetilde{D}$ such that $0 \leq y<\widetilde{f}(\nu, k, m)$. If $\left(c_{i}^{*}, l_{i}^{*}\right)_{i \in I}$ is a solution to the static maximization problem $(S(\nu))$, then $c_{i}^{*}>0$ and $l_{i}^{*} \in(0,1)$ for any $i \in I$.

iii) For any $(\nu, k, y) \in(0,1] \times \operatorname{int} \widetilde{D}$ :

$$
\frac{\partial \widetilde{V}_{t}(\nu, \lambda, \zeta, k, y)}{\partial k \partial y}>0
$$

That is, for any $\nu \in(0,1], \widetilde{V}_{t}$ is supermodular in the interior of $\widetilde{D}$.

iv) For any $T \geq 0$, the value function $\widetilde{W}_{T}(\nu, \zeta, k)$ is strictly increasing, strictly concave in $k$, nonnegative with $W_{T}(\nu, \zeta, k)>0$ for $k>0$ and continuous at any $(\nu, k) \in(0,1] \times \mathbb{R}_{+}$. Moreover,

$$
\lim _{\nu \rightarrow 0} \widetilde{W}_{T}(\nu, \zeta, k)=W_{T}(\zeta, k) .
$$

v) The function $\widetilde{\varphi}$ is continuous at any $(\nu, k) \in[0,1] \times \mathbb{R}_{+}$and therefore uniformly continuous at any $(\nu, k) \in[0,1] \times\left[0, A\left(k_{0}\right)\right]$. Moreover,

$$
\lim _{\nu \rightarrow 0} \widetilde{\varphi}_{T}(\nu, \zeta, k)=\varphi_{T}(\zeta, k) .
$$


Proof: The strict concavity of $\widetilde{V}_{t}$ is standard. Continuity follows from the Maximum Theorem. The proof of claim (ii) is given below. To prove claim (iii) we need the following two results.

Claim 1 Let

$$
V=\left(\begin{array}{ccccc}
a_{1}+b & b & \cdots & b & b \\
b & a_{2}+b & \ldots & \ldots & b \\
\ldots & \ldots & \vdots & \ldots & \vdots \\
b & b & \cdots & a_{q-1}+b & b \\
b & b & \cdots & b & a_{q}+b
\end{array}\right)
$$

with $b<0, a_{i}<0, \forall i=1, \ldots, q$. Then $V^{-1}$ is negative definite and $V^{-1} \mathbf{1}<\mathbf{0}$.

Proof: It is easy to show that $V$ is negative definite. Hence, $V^{-1}$ exists and it is negative definite. Let $\mathbf{x}=V^{-1} \mathbf{1}$. Since $V \mathbf{x}=\mathbf{1}$, it follows that

$$
a_{i} x_{i}+b \sum_{i=1}^{q} x_{i}=1, \forall i .
$$

This in turn implies that $x_{1}=\frac{a_{i}}{a_{1}} x_{i}, \forall i \neq 1$. If $x_{1} \geq 0$, then $x_{i} \geq 0, \forall i \neq 1$ while $a_{i} x_{i}+b \sum_{i=1}^{q} x_{i} \leq 0$ : a contradiction. Hence, $x_{i}<0, \forall i=1, \ldots, q$.

Claim 2 Let $A$ be a $N \times N$ symmetric matrix. Given $r=1, \ldots, N$, denote by ${ }_{r} A$ the $r \times N$ submatrix where only the $r$ rows are retained and by ${ }_{r} A_{r}$ the $r \times r$ submatrix where only the first $r \leq N$ rows and $r \leq N$ columns are retained. Let $B$ be an $N \times S$ submatrix with $S \leq N$ and rank equal to $S$. $A$ is negative definite on $\left\{\mathbf{z} \in \mathbb{R}^{N}: B \mathbf{z}=0\right\}$ (i.e $\mathbf{z}^{T} A \mathbf{z}<0, \forall \mathbf{z} \in \mathbb{R}^{N}$ with $B \mathbf{z}=0$ and $\mathbf{z} \neq 0$ ) if and only if

$$
(-1)^{r}\left|\begin{array}{cc}
{ }_{r} A_{r} & { }_{r} B \\
\left({ }_{r} B\right)^{T} & 0
\end{array}\right|>0
$$

for $r=S+1, \ldots, N$.

Proof: See Mas-Colell et al. (1995) Theorem M.D.3.

(ii) Let $\left(c_{i}^{*}\right)_{i \in I}=\left(c_{i}\left(\nu, \lambda, \zeta^{t}, k, y\right)\right)_{i \in I},\left(l_{i}^{*}\right)_{i \in I}=\left(l_{i}\left(\nu, \lambda, \zeta^{t}, k, y\right)\right)_{i \in I}$ denote a solution to the maximization problem $S(\nu)$. Since $(k, y) \in \widetilde{D}$ with $0 \leq y<$ $\tilde{f}(\nu, k, m)$, there exists $\varepsilon>0$ such that $0<\varepsilon+y<f(k, m-\varepsilon)+\nu(\# I)(1-$ $\left.\frac{\varepsilon}{\# I}\right)^{\alpha}+\nu(m-\# I)$. Letting $c_{i}=\frac{\varepsilon}{\# I}$ and $l_{i}=\frac{\varepsilon}{\# I}, \forall i \in I$, the Slater condition is satisfied. Hence, there exists Lagrange multipliers $\mu_{t}\left(\nu, \lambda, \zeta^{t}, k, y\right) \in \mathbb{R}$ associated with the constraint $\sum_{i} c_{i}+y \leq \widetilde{f}\left(\nu, k,\left(1-l_{i}\right)_{i \in I}\right)$ and $\eta_{i, t}\left(\nu, \lambda, \zeta^{t}, k, y\right) \in \mathbb{R}$ 
associated with the constraints $l_{i} \leq 1$ such that $\left(\left(c_{i}^{*}\right)_{i \in I},\left(l_{i}^{*}\right)_{i \in I}, \mu_{t},\left(\eta_{i, t}\right)_{i \in I}\right)$ maximizes the associated Lagrangian :

$$
\mathcal{L}=\sum_{i \in I} \lambda_{i}\left(\frac{\beta_{i}}{\beta}\right)^{t} u^{i}\left(c_{i}, l_{i}\right)-\mu_{t}\left[\sum_{i \in I} c_{i}+y-\widetilde{f}\left(\nu, k,\left(1-l_{i}\right)_{i \in I}\right)\right]-\sum_{i \in I} \eta_{i, t}\left(l_{i}-1\right) .
$$

From the The Kuhn-Tucker first-order conditions we get:

$$
\begin{gathered}
\lambda_{i}\left(\frac{\beta_{i}}{\beta}\right)^{t} u_{c}^{i}\left(c_{i}^{*}, l_{i}^{*}\right)-\mu_{t}=0, \forall i \in I \\
\lambda_{i}\left(\frac{\beta_{i}}{\beta}\right)^{t} u_{l}^{i}\left(c_{i}^{*}, l_{i}^{*}\right)-\mu_{t}\left[f_{L}\left(k, m-\sum_{j \in I} l_{j}^{*}\right)+\nu \alpha\left(1-l_{i}^{*}\right)^{\alpha-1}\right]-\eta_{i, t}=0, \forall i \in I \\
\mu_{t} \geq 0, \mu_{t}\left[\sum_{i \in I} c_{i}^{*}+y-f\left(k, m-\sum_{i \in I} l_{i}^{*}\right)-\nu \sum_{i \in I}\left(1-l_{i}^{*}\right)^{\alpha}-\nu(m-\# I)\right]=0 \\
\eta_{i, t} \geq 0, \eta_{i, t}\left(l_{i}^{*}-1\right)=0, \forall i \in I .
\end{gathered}
$$

Since $u^{i}$ is strictly increasing, $u^{i}(0,0)=0$ and $u^{i}$ satisfies the Inada conditions, it follows that $c_{i}^{*}>0$ and $l_{i}^{*}>0, \forall i \in I$. Therefore, $\mu_{t}>0$. Moreover, we have that $l_{i}^{*}<1$ and $\eta_{i, t}=0, \forall i \in I$. The first-order conditions become:

$$
\begin{gathered}
\lambda_{i}\left(\frac{\beta_{i}}{\beta}\right)^{t} u_{c}^{i}\left(c_{i}^{*}, l_{i}^{*}\right)-\mu_{t}=0, \forall i \in I \\
\lambda_{i}\left(\frac{\beta_{i}}{\beta}\right)^{t} u_{l}^{i}\left(c_{i}^{*}, l_{i}^{*}\right)-\mu_{t}\left[f_{L}\left(k, m-\sum_{j \in I} l_{j}^{*}\right)+\nu \alpha\left(1-l_{i}^{*}\right)^{\alpha-1}\right]=0, \forall i \in I \\
\sum_{i \in I} c_{i}^{*}+y-f\left(k, m-\sum_{i \in I} l_{i}^{*}\right)-\nu \sum_{i \in I}\left(1-l_{i}^{*}\right)^{\alpha}-\nu(m-\# I)=0
\end{gathered}
$$

Differentiating the above equations and rearranging we get:

$$
\begin{aligned}
& \lambda_{i}\left(\frac{\beta_{i}}{\beta}\right)^{t} u_{c c}^{i} d c_{i}^{*}+\lambda_{i}\left(\frac{\beta_{i}}{\beta}\right)^{t} u_{c l}^{i} d l_{i}^{*}-d \mu_{t}=0, \forall i \in I \\
& \lambda_{i}\left(\frac{\beta_{i}}{\beta}\right)^{t} u_{c l}^{i} d c_{i}^{*}+\left[\lambda_{i}\left(\frac{\beta_{i}}{\beta}\right)^{t} u_{l l}^{i}-\mu_{t} \frac{\nu \alpha(1-\alpha)}{\left(1-l_{i}^{*}\right)^{2-\alpha}}+\mu_{t} f_{L L}\right] d l_{i}^{*} \\
& +\mu_{t} f_{L L} \sum_{j \in I, j \neq i} d l_{j}^{*}-\left[f_{L}+\frac{\nu \alpha}{\left(1-l_{i}^{*}\right)^{1-\alpha}}\right] d \mu_{t}=\mu_{t} f_{k L} d k, \forall i \in I \\
& \sum_{i \in I} d c_{i}^{*}+d y-f_{k} d k+f_{L} \sum_{i \in I} d l_{i}^{*}+\sum_{i \in I} \frac{\nu \alpha}{\left(1-l_{i}^{*}\right)^{1-\alpha}} d l_{i}^{*}=0 .
\end{aligned}
$$

For all $i \in I$ let

$$
p=\mu_{t} f_{L L}, p_{1 i}=\frac{\nu \alpha}{\left(1-l_{i}^{*}\right)^{1-\alpha}}, p_{2 i}=-\mu_{t} \frac{\nu \alpha(1-\alpha)}{\left(1-l_{i}^{*}\right)^{2-\alpha}} .
$$


and

$$
a_{i}=\lambda_{i}\left(\frac{\beta_{i}}{\beta}\right)^{t} u_{c c}^{i}, b_{i}=\lambda_{i}\left(\frac{\beta_{i}}{\beta}\right)^{t} u_{c l}^{i}, c_{i}=\lambda_{i}\left(\frac{\beta_{i}}{\beta}\right)^{t} u_{l l}^{i}+p_{2 i} .
$$

With this notation the first order conditions can be written as follows:

$$
\begin{gathered}
a_{i} d c_{i}^{*}+b_{i} d l_{i}^{*}-d \mu_{t}=0, \forall i \in I \\
b_{i} d c_{i}^{*}+\left[c_{i}+p\right] d l_{i}^{*}+p \sum_{j \in I, j \neq i} d l_{j}^{*}-\left(f_{L}+p_{1 i}\right) d \mu_{t}=\mu_{t} f_{L k} d k, \forall i \in I . \\
\sum_{i \in I} d c_{i}^{*}+\sum_{i \in I}\left(f_{L}+p_{1 i}\right) d l_{i}^{*}=f_{k} d k-d y .
\end{gathered}
$$

Assume for simplicity that $I=\{1, \ldots, q\}$ where $q=\# I$. We can alternatively write these equations in a matrix form, $A X=X_{0}$, where:

$$
\begin{gathered}
A=\left(\begin{array}{ccccccc}
a_{1} & 0 \cdots & 0 & b_{1} & 0 \cdots & 0 & -1 \\
0 & \vdots & \vdots & 0 & \vdots & \vdots & -1 \\
0 & 0 \cdots & a_{q} & 0 & 0 \cdots & b_{q} & -1 \\
b_{1} & 0 \cdots & 0 & c_{1}+p & p \cdots & p & -f_{L}-p_{11} \\
0 & \vdots & 0 & \vdots & \vdots & \vdots & \vdots \\
0 & 0 \cdots & b_{q} & p & p \cdots & c_{q}+p & -f_{L}-p_{1 q} \\
1 & 1 \cdots & 1 & f_{L}+p_{11} & \cdots & f_{L}+p_{1 q} & 0
\end{array}\right) \\
\\
X=\left(\begin{array}{c}
d c_{1}^{*} \\
\vdots \\
d c_{q}^{*} \\
d l_{1}^{*} \\
\vdots \\
d l_{q}^{*} \\
d \mu_{t}
\end{array}\right), X_{0}=\left(\begin{array}{c}
0 \\
\vdots \\
0 \\
\mu_{t} f_{k L} d k \\
\vdots \\
\mu_{t} f_{k L} d k \\
f_{k} d k-d y
\end{array}\right)
\end{gathered}
$$

In particular,

$$
A=\left(\begin{array}{cc}
M & -\mathbf{d}^{T} \\
\mathbf{d} & 0
\end{array}\right)
$$

where:

$$
M=\left(\begin{array}{ll}
M_{11} & M_{12} \\
M_{21} & M_{22}
\end{array}\right), \mathbf{d}=\left(1, \ldots, 1, f_{L}+p_{11}, \ldots, f_{L}+p_{1 q}\right)
$$


and

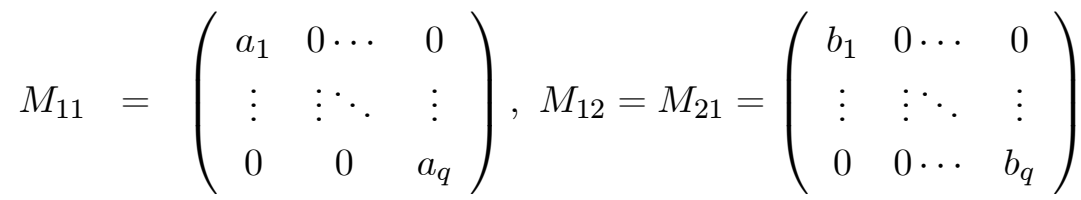

$$
\begin{aligned}
& M_{22}=\left(\begin{array}{ccc}
c_{1}+p & p \cdots & p \\
\vdots & \vdots & \vdots \\
p & p \cdots & c_{q}+p
\end{array}\right) \text {. }
\end{aligned}
$$

We show that $A$ is invertible. Let $\widehat{A}$ be the matrix obtained from $A$ by changing the sign of the last column, i.e

$$
\widehat{A}=\left(\begin{array}{cc}
M & \mathbf{d}^{T} \\
\mathbf{d} & 0
\end{array}\right) .
$$

Observe that $M_{22}=N_{1}+N_{2}$, where

$$
N_{1}=\left(\begin{array}{ccc}
c_{1} & 0 \cdots & 0 \\
\vdots & \vdots & \vdots \\
0 & 0 \cdots & c_{q}
\end{array}\right), N_{2}=\left(\begin{array}{ccc}
p & p \cdots & p \\
\vdots & \vdots & \vdots \\
p & p \cdots & p
\end{array}\right)
$$

Let $\mathbf{z}=\left\{z_{1}, \ldots, z_{q}, \varsigma_{1}, \ldots, \varsigma_{q}\right)$ where $\mathbf{z} \neq \mathbf{0}$. Since $p_{2 i}<0$ and $a_{i} c_{i}-b_{i}^{2} \geq 0$ (since $u^{i}$ is concave), it follows that

$$
\begin{aligned}
\mathbf{z}^{T} M \mathbf{z} & =\mathbf{z}^{T}\left(\begin{array}{cc}
M_{11} & M_{12} \\
M_{21} & N_{1}
\end{array}\right) \mathbf{z}+\mathbf{z}^{T}\left(\begin{array}{cc}
0 & 0 \\
0 & N_{2}
\end{array}\right) \mathbf{z} \\
& =\sum_{i \in I}\left[a_{i}\left(z_{i}+\frac{b_{i}}{a_{i}} \varsigma_{i}\right)^{2}+\frac{a_{i} c_{i}-b_{i}^{2}}{a_{i}} \varsigma_{i}^{2}\right]+p\left(\sum_{i \in I} \varsigma_{i}\right)^{2}<0,
\end{aligned}
$$

Therefore, $M$ is negative definite. Let

$$
B=\left(\begin{array}{c}
\mathbf{d}^{T} \\
0
\end{array}\right) \text {. }
$$

Since $M$ is negative definite on $\left\{\mathbf{z} \in \mathbb{R}^{2 q+1}: B \mathbf{z}=0\right\}$, (i.e $\mathbf{z}^{T} M \mathbf{z}<0, \forall \mathbf{z} \in$ $\mathbb{R}^{2 q+1}$ with $B \mathbf{z}=0$ and $\mathbf{z} \neq 0$ ), it follows from Claim 2 that:

$$
(-1)^{2 q} \operatorname{det}\left(\begin{array}{cc}
2 q M_{2 q} & { }_{2 q} B \\
(2 q B)^{T} & 0
\end{array}\right)=(-1)^{2 q}|\widehat{A}|>0 \text { or }|\widehat{A}|>0 .
$$

Since $|A|=-|\widehat{A}|<0$ the matrix $A$ is invertible. By the Implicit Function Theorem, $c_{i}\left(\nu, \zeta^{t}, k, y\right), l_{i}\left(\nu, \zeta^{t}, k, y\right)$ and $\mu_{t}\left(\nu, \zeta^{t}, k, y\right)$ are $C^{1}$ in a neighborhood 
of $(k, y) \in$ int $\widetilde{D}$. The Envelope Theorem then implies:

$$
\begin{aligned}
\frac{\partial \widetilde{V}_{t}(\nu, \lambda, \zeta, k, y)}{\partial k} & =\mu_{t} f_{k}\left(k, m-\sum_{i \in I} l_{i}^{*}\right) \\
\frac{\partial \widetilde{V}_{t}(\nu, \lambda, \zeta, k, y)}{\partial y} & =-\mu_{t} .
\end{aligned}
$$

From equations (1),(2),(3) we obtain:

$$
\begin{aligned}
& {\left[\left(f_{L}+p_{11}-\frac{b_{1}}{a_{1}}, . ., f_{L}+p_{1 q}-\frac{b_{q}}{a_{q}}\right) V^{-1}\left(\begin{array}{c}
f_{L}+p_{11}-\frac{b_{1}}{a_{1}} \\
\vdots \\
f_{L}+p_{1 q}-\frac{b_{q}}{a_{q}}
\end{array}\right)+\sum_{i=1}^{q} \frac{1}{a_{i}}\right] d \mu_{t} } \\
= & {\left[-\mu_{t} f_{L k}\left(f_{L}+p_{11}-\frac{b_{1}}{a_{1}}, . ., f_{L}+p_{1 q}-\frac{b_{q}}{a_{q}}\right) V^{-1}\left(\begin{array}{c}
1 \\
\vdots \\
1
\end{array}\right)+f_{k}\right] d k-d y, }
\end{aligned}
$$

with

$$
V=\left(\begin{array}{ccc}
c_{1}-\frac{b_{1}^{2}}{a_{1}}+p & \cdots & p \\
\vdots & \vdots & \vdots \\
p & \cdots & c_{q}-\frac{b_{q}^{2}}{a_{q}}+p
\end{array}\right)
$$

Observe that the matrix $V$ satisfies the hypothesis of Claim 1. Therefore, $V^{-1}$ exists and

$$
V^{-1}\left(\begin{array}{c}
1 \\
\vdots \\
1
\end{array}\right)<\left(\begin{array}{c}
0 \\
\vdots \\
0
\end{array}\right)
$$

From the first-order conditions we have:

$$
f_{L}+p_{1 i}=\frac{u_{l}^{i}\left(c_{i}^{*}, l_{i}^{*}\right)}{u_{c}^{i}\left(c_{i}^{*}, l_{i}^{*}\right)}, \forall i .
$$

Given assumption $\mathbf{U} 4$ we also have:

$$
\begin{aligned}
f_{L}+p_{1 i}-\frac{b_{i}}{a_{i}} & =\frac{u_{l}^{i}}{u_{c}^{i}}-\frac{u_{c l}^{i}}{u_{c c}^{i}} \\
& =\frac{u_{l}^{i}}{u_{c c}^{i}}\left(\frac{u_{c c}^{i}}{u_{c}^{i}}-\frac{u_{c l}^{i}}{u_{l}^{i}}\right) \geq 0 .
\end{aligned}
$$

It follows that:

$$
\frac{\partial \mu_{t}}{\partial k}<0
$$

and in particular

$$
\frac{\partial^{2} \widetilde{V}_{t}(\nu, \lambda, \zeta, k, y)}{\partial k \partial y}=-\frac{\partial \mu_{t}}{\partial k}>0, \forall(k, y) \in \operatorname{int} \widetilde{D}
$$


iv) It follows from standard arguments.

v) For $\nu \in(0,1]$ the claim follows from the Maximum Theorem. When $\nu=0$, then $\widetilde{\varphi}=\varphi$ and Proposition 6 applies. $(\widehat{Q})$.

Equipped with the last results we return to the study of problems $(Q)$ and

Proposition 8 Assume $\boldsymbol{U 1 - U} 4$ and $\boldsymbol{F} 1-\boldsymbol{F} 4$. Then,

i) The functions $V_{t}$ and $\widehat{V}$ are supermodular in the interior of $D$, i.e for $(k, y),\left(k^{\prime}, y^{\prime}\right) \in \operatorname{int} D$ we have:

$$
\begin{aligned}
& V_{t}\left(\lambda, \zeta,(k, y) \vee\left(k^{\prime}, y^{\prime}\right)\right)+V_{t}\left(\lambda, \zeta,(k, y) \wedge\left(k^{\prime}, y^{\prime}\right)\right) \\
\geq & V_{t}(\lambda, \zeta, k, y)+V_{t}\left(\lambda, \zeta, k^{\prime}, y^{\prime}\right)
\end{aligned}
$$

and

$$
\begin{aligned}
& \widehat{V}\left(\lambda,(k, y) \vee\left(k^{\prime}, y^{\prime}\right)\right)+\widehat{V}\left(\lambda,(k, y) \wedge\left(k^{\prime}, y^{\prime}\right)\right) \\
\geq & \widehat{V}(\lambda, k, y)+\widehat{V}\left(\lambda, k^{\prime}, y^{\prime}\right) .
\end{aligned}
$$

ii) The policy function $\varphi(\underset{\sim}{0}, k)$ is non-decreasing in $k$. As a consequence, the optimal capital path associated with problem $\widehat{Q}$ is monotonic. Moreover, if $k_{0} \leq k_{0}^{\prime}$ and $\mathbf{k}^{*}, \mathbf{k}^{\prime}$ are the optimal paths starting respectively from $k_{0}$ and $k_{0}^{\prime}$, then $k_{t}^{*} \leq k_{t}^{\prime}, \forall t$.

Proof: i) Let $(k, y) \in$ intD. Observe that for any $\nu \in[0,1]$ we have $0<y<$ $\widetilde{f}(\nu, k, m)$. From Proposition 7 , given $(k, y),\left(k^{\prime}, y^{\prime}\right) \in \operatorname{int} D$, it follows that:

$$
\begin{aligned}
& \widetilde{V}_{t}\left(\nu, \lambda, \zeta,(k, y) \vee\left(k^{\prime}, y^{\prime}\right)\right)+\widetilde{V}_{t}\left(\nu, \lambda, \zeta,(k, y) \wedge\left(k^{\prime}, y^{\prime}\right)\right) \\
\geq & \widetilde{V}_{t}(\nu, \lambda, \zeta, k, y)+\widetilde{V}_{t}\left(\nu, \lambda, \zeta, k^{\prime}, y^{\prime}\right) .
\end{aligned}
$$

Taking the limits of both sides as $\nu \rightarrow 0$ we get:

$$
\begin{aligned}
& V_{t}\left(\lambda, \zeta,(k, y) \vee\left(k^{\prime}, y^{\prime}\right)\right)+V_{t}\left(\lambda, \zeta,(k, y) \wedge\left(k^{\prime}, y^{\prime}\right)\right) \\
\geq \quad & V_{t}(\lambda, \zeta, k, y)+V_{t}\left(\lambda, \zeta, k^{\prime}, y^{\prime}\right) .
\end{aligned}
$$

That is, $V_{t}$ is supermodular on the interior of $D$. The supermodularity of $\widehat{V}$ follows from the fact that $V_{t}=\widehat{V}$ in case where all agents have the same discount factor, i.e $\beta_{i}=\beta, \forall i \in I$.

ii) Recall that problem $\widehat{Q}$ is stationary. Assume that $k_{0}<k_{0}^{\prime}$. If $k_{0}=0$, then $\varphi\left(\underset{\sim}{0}, k_{0}\right)=0$ and $\varphi\left(\underset{\sim}{0}, k_{0}^{\prime}\right)>0$. Let $k_{0}>0$ and assume the contrary, i.e $\varphi\left(\underset{\sim}{0}, k_{0}\right)>\varphi\left(\underset{\sim}{0}, k_{0}^{\prime}\right)$. We consider two cases:

Case 1: Assume that $\varphi\left(\underset{\sim}{0}, k_{0}\right)<f\left(k_{0}, m\right)$. 
Observe that

$$
\begin{aligned}
& 0<\varphi\left(\underset{\sim}{0}, k_{0}^{\prime}\right)<f\left(k_{0}, m\right) \\
& 0<\varphi\left(\underset{\sim}{0}, k_{0}\right)<f\left(k_{0}^{\prime}, m\right) .
\end{aligned}
$$

That is, $\varphi\left(\underset{\sim}{\underset{\sim}{0}}, k_{0}^{\prime}\right)$ is feasible from $k_{0}$ and $\varphi\left(\underset{\sim}{0}, k_{0}\right)$ is feasible from $k_{0}^{\prime}$. Since $\widehat{V}$ is supermodular in the interior of $D$, the claim follows from standard arguments (see Majumdar et al. (2000), Ch. 2, Proposition 5.2).

Case 2: Assume that $\varphi\left(\underset{\sim}{0}, k_{0}\right)=f\left(k_{0}, m\right)$.

We have that $0<\varphi\left(\underset{\sim}{0}, k_{0}^{\prime}\right)<f\left(k_{0}^{\prime}, m\right)$. The continuity of $\varphi$ implies that for $k<k_{0}^{\prime}$ with $k$ sufficiently close to $k_{0}^{\prime}$ we have $0<\varphi(\underset{\sim}{0}, k)<f(k, m)$. Let $k^{1} \in\left[k_{0}, k_{0}^{\prime}\right)$ be the first element, such that, $\varphi\left(\underset{\sim}{0}, k^{1}\right)=f\left(k^{1}, m\right)$. For any $k \in\left(k^{1}, k_{0}^{\prime}\right]$, we have $0<\varphi(\underset{\sim}{0}, k)<f(k, m)$ and $\varphi(\underset{\sim}{0}, k) \leq \varphi\left(\underset{\sim}{0}, k_{0}^{\prime}\right)$ (refer to Case 1). It follows that

$$
\varphi\left(\underset{\sim}{0}, k_{0}^{\prime}\right) \geq \varphi\left(\underset{\sim}{0}, k^{1}\right)=f\left(k^{1}, m\right) \geq f\left(k_{0}, m\right)=\varphi\left(\underset{\sim}{0}, k_{0}\right) .
$$

We conclude the proof.

Lemma 4 Let $k_{0}>0$. Under Assumptions U1-U4, F1-F4, if $\mathbf{k}^{*}$ is the optimal path starting from $k_{0}$, then $k_{t}^{*}>0, \forall t$.

Proof: See Appendix A.

To proceed further we need to impose some additional structure on preferences and production technology.

Assumption U5: For any period utility function $u^{i}$ that satisfies $u^{i}(c, 0)=$ $u^{i}(0, l)=0, \forall c \geq 0, l \geq 0$ we assume that $u_{c}^{i}(x, x)$ and $u_{l}^{i}(x, x)$ are nonincreasing in $x$

Remark 3 Assumption $\boldsymbol{U} 5$ is satisfied for the Cobb-Douglas class $u(c, l)=$ $c^{\alpha} l^{\beta}$, with $\alpha+\beta \leq 1$, as well as, the CES class $u(c, l)=\left[c^{\alpha}+l^{\alpha}\right]^{\frac{1}{\alpha}}$, with $\alpha<0$. It is also satisfied by the class of period utilities $u(c, l)=m[h(c, l)]$, where $h$ is a homogeneous function of degree 1 satisfying $h(c, 0)=h(0, l)=0$ and $m$ is a monotone increasing and concave transformation with $m(0)=0$.

Assumption F5: The production function $F$ is homogeneous of degree $\alpha \in$ $(0,1]$, i.e $F(k, L)=L^{\alpha} F\left(\frac{k}{L}, 1\right)$.

Lemma 5 Let $k_{0}>0$. Under Assumptions U1-U5, F1-F5, if $\mathbf{k}^{*}$ is the optimal path starting from $k_{0}$, then $k_{t}^{*}$ cannot converge to zero. 
Proof: See Appendix A.

The following result proves the existence of a unique non-trivial steady state for the planner's problem involving only the most patient consumers.

Proposition 9 Let $k_{0}>0$ and $\mathbf{k}^{*}$ denote the optimal solution from $k_{0}$ to problem $(\widehat{Q})$. There exists a unique $k^{s}$ such that $k_{t}^{*} \rightarrow k^{s}$. Moreover let $\left(\mathbf{c}_{i}^{*}, \mathbf{l}_{i}^{*}\right)_{i \in I_{1}}$ denote the individuals' optimal intertemporal allocations associated with $\mathbf{k}^{*}$. There exists $\left(c_{i}, l_{i}\right)_{i \in I_{1}}$ such that $c_{i, t}^{*} \rightarrow c_{i}^{s}, l_{i, t}^{*} \rightarrow l_{i}^{s}, \forall i \in I_{1}$.

Proof: From Proposition 8(ii) we know that the optimal capital sequence $\mathbf{k}^{*}$ is bounded and monotonic. In addition, Lemma 5 implies that $k_{t}^{*} \rightarrow k^{s}>0$. By the principle of optimality, for any $t$, we have:

$$
\widehat{W}\left(k_{t}^{*}\right)=\widehat{V}\left(\lambda, k_{t}^{*}, k_{t+1}^{*}\right)+\beta \widehat{W}\left(k_{t+1}^{*}\right)
$$

Taking the limits as $t \rightarrow+\infty$ we obtain:

$$
\widehat{W}\left(k^{s}\right)=\widehat{V}\left(\lambda, k^{s}, k^{s}\right)+\beta \widehat{W}\left(k^{s}\right) .
$$

It follows that $k^{s}$ is a steady state. From Proposition 1 , there exists $\left(c_{i}^{s}, l_{i}^{s}\right)_{i \in I_{1}}$ associated with $k^{s}$ that solves:

$$
\begin{aligned}
\widehat{V}\left(\lambda, k^{s}, k^{s}\right)=\max & \sum_{i \in I_{1}} \lambda_{i} u_{i}\left(c_{i}, l_{i}\right) \\
\text { s.t. } & \sum_{i \in I_{1}} c^{i}+k^{s} \leq f\left(k^{s}, m-\sum_{i \in I_{1}} l^{i}\right) \\
& c^{i} \geq 0, l^{i} \in[0,1], \forall i \in I_{1} .
\end{aligned}
$$

Observe that $0<k^{s}<f\left(k^{s}, m\right)$. Indeed, if $k^{s}=f\left(k^{s}, m\right)$, then:

$$
\sum_{i \in I_{1}} c_{i}^{s}=0 \text { and } \sum_{i \in I_{1}} l_{i}^{s}=0 .
$$

This implies that $\widehat{V}\left(\lambda, k^{s}, k^{s}\right)=0$. Since $\widehat{W}\left(k^{s}\right)>0$ for $k^{s}>0$ (see Proposition $4)$ and $\beta \in(0,1)$, we obtain a contradiction.

From Proposition 2 we have $c_{i}^{s}=c_{i}\left(\lambda, \underset{\sim}{\underset{0}{0}}, k^{s}, k^{s}\right)>0$ and $l_{i}^{s}=l_{i}\left(\lambda, \underset{\sim}{0}, k^{s}, k^{s}\right)>$ $0, \forall i \in I_{1}$. Moreover, since $c_{i}\left(\lambda, \underset{\sim}{0}, k_{t}^{*}, k_{t+1}^{*}\right)$ and $l_{i}\left(\lambda, \underset{\sim}{0}, k_{t}^{*}, k_{t+1}^{*}\right)$ are continuous functions and $k_{t}^{*} \rightarrow k^{s}$, it follows that for any $i \in I_{1}$ :

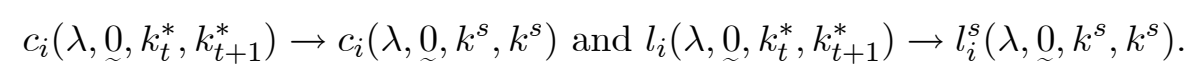

The proof of uniqueness of steady state is given in Appendix B. 
Remark 4 We provide below an example of an economy having a steady state in which some agent $i$ does not provide labor, i.e an economy in which $l_{i}^{s}=1$ for some agent $i$. The example illustrates in a clear way the possibility of corner solutions in our model. In the example, one of the consumers strongly prefers leisure. It is therefore optimal for the social planner to allocate to this consumer the total endowment of his leisure time.

Example 1 Consider an economy with 2 agents having period utilities functions of the following form: $u^{1}\left(c_{1}, l_{1}\right)=c_{1}^{\alpha}+l_{1}^{\alpha}$ and $u^{2}\left(c_{2}, l_{2}\right)=c_{2}^{b}+\theta l_{2}^{b}$, $\alpha, b \in(0,1)$. Technology is Cobb-Douglas, $F(k, L)=k^{\gamma} L^{1-\gamma}$, where $\gamma \in(0,1)$. For simplicity assume that $\delta=1$ (full depreciation). Assume that $\beta_{1}=\beta_{2}=\beta$ (both consumers have the same discount factor, i.e $I_{2}$ is an empty set) and that the social planner puts equal weights to both consumers, $\lambda_{1}=\lambda_{2}=\frac{1}{2}$. To simplify further, assume that $\alpha=b$.

It is easy to check that the steady state of this economy is described by the solution of the following system of equations:

$$
\begin{aligned}
& c_{1}^{\alpha-1}=c_{2}^{\alpha-1} \\
& \left(\frac{c_{1}}{l_{1}}\right)^{1-\alpha}=(1-\gamma) x^{\gamma}+\eta_{1} \\
& \theta\left(\frac{c_{2}}{l_{2}}\right)^{1-\alpha}=(1-\gamma) x^{\gamma}+\eta_{2} \\
& x=(\gamma \beta)^{\frac{1}{1-\gamma}} \\
& k=x\left(2-l_{1}-l_{2}\right) \\
& c_{1}+c_{2}-\left(2-l_{1}-l_{2}\right)\left[x^{\gamma}-x\right]=0
\end{aligned}
$$

The constant $\eta_{i}$ denotes the multiplier associated with the constraint $l_{i} \leq 1$. From equations (1)-(4) it follows that

$$
\begin{aligned}
& c_{1}=c_{2}=l_{1}\left[(1-\gamma) x^{\gamma}+\eta_{1}\right]^{\frac{1}{1-\alpha}} \\
& l_{2}=\left[\frac{\theta l_{1}^{1-\alpha}\left[(1-\gamma) x^{\gamma}+\eta_{1}\right]}{\left.(1-\gamma) x^{\gamma}+\eta_{2}\right]}\right]^{\frac{1}{1-\alpha}} .
\end{aligned}
$$

One can show that the system of steady state equations (1)-(8) is consistent with a solution $\left(\left(c_{i}^{s}, l_{i}^{s}\right)_{i=1,2}, x^{s}, k^{s}, \eta_{1}^{s}, \eta_{2}^{s}\right)$ such that $l_{2}^{s}=1$. Indeed, assume that $\left(\left(c_{i}^{s}, l_{i}^{s}\right)_{i=1,2}, x^{s}, k^{s}, \eta_{1}^{s}, \eta_{2}^{s}\right)$ is a solution such that $l_{2}^{s}=1$. Equation (4) gives the value of $x^{s}$. From equations (6)-(7) we have that

$$
2 l_{1}^{s}\left[(1-\gamma)\left(x^{s}\right)^{\gamma}+\eta_{1}^{s}\right]^{\frac{1}{1-\alpha}}=\left(1-l_{1}^{s}\right)\left[\left(x^{s}\right)^{\gamma}-x^{s}\right] .
$$

For any $\eta_{1}^{s} \geq 0$ equation (9) is satisfied for a unique $l_{1}^{s} \in(0,1)$. In this case, we have $\eta_{1}^{s}=0$. From equation (8)

$$
l_{1}^{s}\left[\theta(1-\gamma)\left(x^{s}\right)^{\gamma}\right]^{\frac{1}{1-\alpha}}=(1-\gamma)\left(x^{s}\right)^{\gamma}+\eta_{2}^{s} .
$$


Let $\bar{\theta}$ such that $l_{1}^{s}\left[\bar{\theta}(1-\gamma) x^{\gamma}\right]^{\frac{1}{1-\alpha}}=(1-\gamma) x^{\gamma}$. For any $\theta>\bar{\theta}$ equation (10) determines $\eta_{2}^{s}>0$. Given $l_{1}^{s}$, equation (7) determines $c_{1}^{s}$ and $c_{2}^{s}$. Finally, $k^{s}$ is determined by equation (5).

We have the following result.

Lemma 6 Let $a \in(0, \bar{k}]$. Then, for any $\varepsilon>0$, there exists $T(a, \varepsilon)$, such that, for any $k \geq a$, for any $t \geq T(a, \varepsilon)$ we have:

$$
\left|\varphi^{t}(\underset{\sim}{0}, k)-k^{s}\right|<\varepsilon
$$

Proof: Choose some $a \in(0, \bar{k}]$. Let $\varepsilon>0$ and $k \geq a$. Given Proposition 9, there exists $T(k, \varepsilon)$ such that ,for any $t \geq T(k, \varepsilon)$, we have:

$$
\left|\varphi^{t}(\underset{\sim}{0}, k)-k^{s}\right|<\varepsilon
$$

Since $\varphi^{t} \underset{\sim}{\underset{\sim}{0}, k)}$ is continuous, there exists a neighborhood $\mathcal{N}(k)$ of $k$, such that, for any $k^{\prime} \in \mathcal{N}(k)$ we have:

$$
\left|\varphi^{T(k, \varepsilon)}\left(\underset{\sim}{0}, k^{\prime}\right)-k^{s}\right|<\varepsilon
$$

Assume that $k<k^{s}$. Since $\left(\varphi^{t}(\underset{\sim}{0}, k)\right)$ is a non-decreasing sequence, it follows that for any $k^{\prime} \in \mathcal{N}(k)$, for any $t \geq T(k, \varepsilon)$ we have:

$$
\begin{aligned}
\left|\varphi^{t}\left(\underset{\sim}{0}, k^{\prime}\right)-k^{s}\right| & =k^{s}-\varphi^{t}\left(\underset{\sim}{0}, k^{\prime}\right) \\
& \leq k^{s}-\varphi^{T(k, \varepsilon)}{\left.\underset{\sim}{0}, k^{\prime}\right)}^{<} .
\end{aligned}
$$

When $k \geq k^{s}$ the argument is similar. Consider next a finite covering $\left(\mathcal{N}\left(k_{j}\right)\right)_{j=1}^{J}$ of $[a, \bar{k}]$ and let $T(a, \varepsilon)=\max _{j \in\{1, \ldots, J\}}\left\{T\left(k_{j}, \varepsilon\right)\right\}$.

We now return to the initial problem involving all agents, i.e problem $(Q)$. The following Proposition shows that the optimal capital sequence is bounded away from zero.

Proposition 10 For any $k_{0}>0$ and $\mathbf{k}^{*}$ optimal from $k_{0}$, there exists $\gamma>0$ such that $k_{t}^{*} \geq \gamma, \forall t$.

Proof: Lemma 4 implies that there exists $a \in\left(0, k^{s}\right)$ and a subsequence $\left(k_{T_{n}}^{*}\right)_{n \in \mathbb{N}}$ such that $k_{T_{n}}^{*} \geq a, \forall n \in \mathbb{N}$. Choose $\varepsilon>0$ such that $a-\varepsilon>0$ 
and $k^{s}-2 \varepsilon>a$. Let $T(a, \varepsilon)$ be as in Lemma 6 . It follows that for any $n \in \mathbb{N}$ we have:

$$
\left|\varphi^{T(\varepsilon, a)}\left(\underset{\sim}{0}, k_{T_{n}}^{*}\right)-k^{s}\right|<\varepsilon .
$$

This in turn implies that

$$
\varphi^{T(\varepsilon, a)}\left(\underset{\sim}{0}, k_{T_{n}}^{*}\right)>k^{s}-\varepsilon>a-\varepsilon .
$$

Let $t \in\{1, \ldots, T(a, \varepsilon)\}$. The uniform continuity of $\varphi^{t}$ implies that for $n$ large enough we have:

$$
\left|\varphi^{t}\left(\zeta^{T_{n}}, k_{T_{n}}^{*}\right)-\varphi^{t}\left(\underset{\sim}{0}, k_{T_{n}}^{*}\right)\right|<\varepsilon
$$

It follows that

$$
\varphi^{t}\left(\zeta^{T_{n}}, k_{T_{n}}^{*}\right)>\varphi^{t}\left(\underset{\sim}{0}, k_{T_{n}}^{*}\right)-\varepsilon .
$$

Since $a \in\left(0, k^{s}\right)$, we have that $\varphi^{t}(\underset{\sim}{0}, a) \geq a, \forall t$. Moreover, $k_{T_{n}}^{*} \geq a$ implies that $\varphi^{t}\left(\underset{\sim}{0}, k_{T_{n}}^{*}\right) \geq \varphi^{t}(\underset{\sim}{0}, a) \geq a, \forall t$. Therefore, for any $t \in\{1, \ldots, T(a, \varepsilon)\}$, it follows from (2) that:

$$
\varphi^{t}\left(\zeta^{T_{n}}, k_{T_{n}}^{*}\right)>a-\varepsilon .
$$

In particular, for $t=T(\varepsilon, a)$, the inequalities (1), (2) imply that:

$$
\varphi^{T(a, \varepsilon)}\left(\zeta^{T_{n}}, k_{T_{n}}^{*}\right)>k^{s}-2 \varepsilon>a .
$$

By definition:

$$
\varphi^{T(a, \varepsilon)}\left(\zeta^{T_{n}}, k_{T_{n}}^{*}\right)=k_{T_{n}+T(a, \varepsilon)}^{*}>a .
$$

In a similar way, Lemma 6 implies that for any $n \in \mathbb{N}$ :

$$
\left|\varphi^{T(a, \varepsilon)}\left(\underset{\sim}{0}, k_{T_{n}+T(a, \varepsilon)}^{*}\right)-k^{s}\right|<\varepsilon .
$$

Moreover, for any $t \in\{1, \ldots, T(a, \varepsilon)\}$, the uniform continuity of $\varphi^{t}$ implies that:

$$
\left|\varphi^{t}\left(\zeta^{T_{n}+T(a, \varepsilon)}, k_{T_{n}+T(a, \varepsilon)}^{*}\right)-\varphi^{t}\left(\underset{\sim}{0}, k_{T_{n}+T(a, \varepsilon)}^{*}\right)\right|<\varepsilon .
$$

It follows that for any $t \in\{1, \ldots, T(a, \varepsilon)\}$ we have:

$$
\varphi^{t}\left(\zeta^{T_{n}+T(a, \varepsilon)}, k_{T_{n}+T(a, \varepsilon)}^{*}\right)>a-\varepsilon
$$

and in particular

$$
\varphi^{T(a, \varepsilon)}\left(\zeta^{T_{n}+T(a, \varepsilon)}, k_{T_{n}+T(a, \varepsilon)}^{*}\right)>k^{s}-2 \varepsilon>a .
$$

By definition, for any $t \in\{1, \ldots, T(a, \varepsilon)\}$, we have:

$$
\varphi^{t}\left(\zeta^{T_{n}+T(a, \varepsilon)}, k_{T_{n}+T(a, \varepsilon)}^{*}\right)=k_{T_{n}+T(a, \varepsilon)+t}^{*} .
$$


This in turn implies that:

$$
\varphi^{t}\left(\zeta^{T_{n}}, k_{T_{n}}^{*}\right)>a-\varepsilon, \forall t=1, \ldots, 2 T(a, \varepsilon) .
$$

Repeating the above argument one can establish that:

$$
\varphi^{t}\left(\zeta^{T_{n}}, k_{T_{n}}^{*}\right)>a-\varepsilon, \forall t=1, \ldots, \infty .
$$

The claim is true for

$$
\gamma=\min \left\{k_{1}^{*}, \ldots, k_{T_{n}}^{*}, a-\varepsilon\right\}
$$

Proposition 11 Let $k_{0}>0$ and $\left(\left(\mathbf{c}_{i}^{*}, \mathbf{l}_{i}^{*}\right)_{i}, \mathbf{k}^{*}\right)$ denote the solution to problem $(P)$. Let $\left(\left(c_{i}^{s}, l_{i}^{s}\right)_{i \in I_{1}}, k^{s}\right)$ denote the steady state associated with problem $(\widehat{Q})$. Then, i) $k_{t}^{*} \rightarrow k^{s}$, ii) $c_{i, t}^{*} \rightarrow c_{i}^{s}$ and $l_{i, t}^{*} \rightarrow l_{i}^{s}, \forall i \in I_{1}$, iii) $c_{i, t}^{*} \rightarrow 0$ and $l_{i, t}^{*} \rightarrow 0$, $\forall i \in I_{2}$.

Proof: Given the previous results, the proof of (i) parallels the one presented in Le Van-Vailakis (2003, Proposition 4). Since $k_{t}^{*} \rightarrow k^{s}$ and $\left(k^{s}, k^{s}\right) \in \operatorname{int} D$, there exists some $T$ such that $\left(k_{t}^{*}, k_{t+1}^{*}\right) \in$ int $D, \forall t \geq T$. We know that for any $i \in I, c_{i}\left(\lambda, \zeta^{t}, k_{t}^{*}, k_{t+1}^{*}\right)$ and $l_{i}\left(\lambda, \zeta^{t}, k_{t}^{*}, k_{t+1}^{*}\right)$ are continuous functions in $[0,1]^{\# I_{2}} \times$ int $D$ and that $V\left(\lambda, \zeta^{t}, k_{t}^{*}, k_{t+1}^{*}\right) \rightarrow \widehat{V}\left(\lambda, k^{s}, k^{s}\right)$. This proves claims (ii) and (iii).

Remark 5 The last Proposition shows that the equilibrium paths associated with problem $(P)$ converge to a limit point. This limit point is the steady state associated with the planner's problem involving only the most patient consumers. The model exhibits the well known property of "the emergence of a dominant consumer" found in the seminal papers of Becker (1980) and Bewley (1982). After all, one can ask if the convergence point is itself a steady state for the problem involving all agents. It is easy to show that this is not true.

Assume that $I_{2} \neq \emptyset$, i.e there exists some $j \in I$ such that $\beta_{j}<\beta$. Let $k^{\text {s be }}$ the steady state associated with problem $(\widehat{Q})$. Let $k_{0}=k^{s}$ and assume that for any $t \geq 1$, (i) $k_{t}^{*}=k^{s}$, (ii) $c_{i, t}^{*}=c_{i}^{s}, l_{i, t}^{*}=l_{i}^{s}, \forall i \in I_{1}$, (iii) $c_{i, t}^{*}=0, l_{i, t}^{*}=0$, $\forall i \in I_{2}$. Since $0<k^{s}<f\left(k^{s}, m\right)$, from Proposition $2(i v)$ it follows that $c_{j, t}^{*}>0$, $l_{j, t}^{*}>0, \forall t \geq 0$. This in turn contradicts the optimality of $k_{t}^{*}=k^{s}$ and $c_{i, t}^{*}=0$, $l_{i, t}^{*}=0, \forall i \in I_{2}$.

In this case, if the economy starts at $k_{0}=k^{s}$ any optimal path $\left(k_{t}^{*}\right)$ converges to $k^{s}$ with $k_{1}^{*} \neq k^{s}$. As a result, the optimal path may exhibit fluctuations at least for the beginning periods. 


\section{Appendix A}

Proof of Lemma 4: We prove the claim for the case where $\# I \geq 2^{3}$. Let $k_{0}>0$ but assume that $k_{1}^{*}=0$. Recall that $L_{t}^{*}=m-\sum_{i \in I} l_{i, t}^{*}$. Since $f\left(k_{0}, L_{0}^{*}\right) \geq(1-\delta) k_{0}>0$, there exists some $i_{1} \in I$ such that $c_{i_{1}, 0}^{*}>0$. Choose $\varepsilon>0$ such that $c_{i_{1}, 0}^{*}>\varepsilon+\varepsilon^{2}$. The proof follows in two steps.

Step 1: We claim that $0 \leq k_{2}^{*}<f(0, m)$.

Assume the contrary. In this case, $c_{i, 1}^{*}=0$ and $l_{i, 1}^{*}=0, \forall i \in I$. Let $\alpha=\frac{\varepsilon+1}{\beta_{i_{1}}}$ and $\gamma=\frac{\varepsilon+1}{\beta_{i_{1}}\left[c_{i_{1}, 0}^{*}-\left(\varepsilon+\varepsilon^{2}\right)\right]}$. Consider the alternative path $\left(\left(\mathbf{c}_{i}, \mathbf{l}_{i}\right)_{i}, \mathbf{k}\right)$ defined as follows:

i) $\quad c_{i_{1}, 0}=c_{i_{1}, 0}^{*}-\left(\varepsilon+\varepsilon^{2}\right), c_{i_{2}, 0}=c_{i_{2}, 0}^{*}+\varepsilon^{2}, c_{i, 0}=c_{i, 0}^{*}, \quad \forall i \in I \backslash\left\{i_{1}, i_{2}\right\}$

ii) $\quad c_{i_{1}, 1}=\alpha \varepsilon, c_{i_{2}, 1}=\varepsilon, c_{i, 1}=0, \forall i \in I \backslash\left\{i_{1}, i_{2}\right\}$

iii) $l_{i, 0}=l_{i, 0}^{*}, \forall i \in I, l_{i_{1}, 1}=\gamma \varepsilon, l_{i_{2}, 1}=\varepsilon, l_{i, 1}=0, \forall i \in I \backslash\left\{i_{1}, i_{2}\right\}$

iv) $c_{i, t}=c_{i, t}^{*}$ and $l_{i, t}=l_{i, t}^{*}, \forall i \in I, \forall t \geq 2$

v) $k_{1}=\varepsilon, k_{t}=k_{t}^{*}, \forall t \geq 2$.

Observe that

$$
c_{i_{1}, 0}^{*}-\left(\varepsilon+\varepsilon^{2}\right)+c_{i_{2}, 0}^{*}+\varepsilon^{2}+\sum_{i \in I \backslash\left\{i_{1}, i_{2}\right\}} c_{i, 0}^{*}+\varepsilon=f\left(k_{0}, L_{0}^{*}\right) .
$$

Moreover,

$$
\begin{aligned}
& f\left(k_{1}, m-l_{i_{1}, 1}-l_{i_{2}, 1}\right)-k_{2}-c_{i_{1}, 1}-c_{i_{2}, 1} \\
= & f(\varepsilon, m-\gamma \varepsilon-\varepsilon)-f(0, m)-\alpha \varepsilon-\varepsilon \\
\geq & \varepsilon\left[f_{k}(\varepsilon, m-\gamma \varepsilon-\varepsilon)-f_{L}(\varepsilon, m-\gamma \varepsilon-\varepsilon)(1+\gamma)-\alpha-1\right] .
\end{aligned}
$$

Due to the Inada conditions on $F$, the term inside the bracket is strictly positive for $\varepsilon$ small enough. This proves feasiblity of the alternative path.

Observe that as $\varepsilon \rightarrow 0$ both $\alpha$ and $\gamma$ converge to a finite value. In addition, $\frac{\alpha}{\gamma}=c_{i_{1}, 0}$. Define:

$$
\begin{aligned}
& \Delta(\varepsilon)=\sum_{i \in I} \lambda_{i} \sum_{t=0}^{\infty} \beta_{i}^{t} u^{i}\left(c_{i, t}, l_{i, t}\right)-\sum_{i \in I} \lambda_{i} \sum_{t=0}^{\infty} \beta_{i}^{t} u^{i}\left(c_{i, t}^{*}, l_{i, t}^{*}\right) \\
= & \lambda_{i_{1}}\left[u^{i_{1}}\left(c_{i_{1}, 0}, l_{i_{1}, 0}\right)-u^{i_{1}}\left(c_{i_{1}, 0}^{*}, l_{i_{1}, 0}^{*}\right)\right]+\lambda_{i_{1}} \beta_{i_{1}}\left[u^{i_{1}}\left(c_{i_{1}, 1}, l_{i_{1}, 1}\right)-u^{i_{1}}\left(c_{i_{1}, 1}^{*}, l_{i_{1}, 1}^{*}\right)\right] \\
+ & \lambda_{i_{2}}\left[u^{i_{2}}\left(c_{i_{2}, 0}, l_{i_{2}, 0}\right)-u^{i_{2}}\left(c_{i_{2}, 0}^{*}, l_{i_{2}, 0}^{*}\right)\right]+\lambda_{i_{2}} \beta_{i_{2}}\left[u^{i_{2}}\left(c_{i_{2}, 1}, l_{i_{2}, 1}\right)-u^{i_{2}}\left(c_{i_{2}, 1}^{*}, l_{i_{2}, 1}^{*}\right)\right] \\
= & \Delta_{i_{1}}(\varepsilon)+\Delta_{i_{2}}(\varepsilon) .
\end{aligned}
$$

The concavity of $u^{i_{1}}$ implies that

$$
\begin{aligned}
\frac{\Delta_{i_{1}}(\varepsilon)}{\lambda_{i_{1}}} & =\beta_{i_{1}}\left[u^{i_{1}}\left(c_{i_{1}, 1}, l_{i_{1}, 1}\right)-u^{i_{1}}\left(c_{i_{1}, 1}^{*}, l_{i_{1}, 1}^{*}\right)\right]+\left[u^{i_{1}}\left(c_{i_{1}, 0}, l_{i_{1}, 0}\right)-u^{i_{1}}\left(c_{i_{1}, 0}^{*}, l_{i_{1}, 0}^{*}\right)\right] \\
& \geq \beta_{i_{1}} u^{i_{1}}(\alpha \varepsilon, \gamma \varepsilon)-u_{c}^{i_{1}}\left(c_{i_{1}, 0}, l_{i_{1}, 0}\right)\left(\varepsilon+\varepsilon^{2}\right) .
\end{aligned}
$$

\footnotetext{
${ }^{3}$ In case where there is only one agent the argument is similar.
} 
If $u_{c l}^{i_{1}}>0$, then

$$
\begin{aligned}
\frac{\Delta_{i_{1}}(\varepsilon)}{\lambda_{i_{1}}} & \geq \beta_{i_{1}} u^{i_{1}}\left(\gamma \varepsilon \frac{\alpha \varepsilon}{\gamma \varepsilon}, \gamma \varepsilon\right)-u_{c}^{i_{1}}\left(c_{i_{1}, 0}, l_{i_{1}, 0}\right)\left(\varepsilon+\varepsilon^{2}\right) \\
& \geq \beta_{i_{1}} u^{i_{1}}\left(\frac{\alpha}{\gamma}, 1\right) \gamma \varepsilon-u_{c}^{i_{1}}\left(c_{i_{1}, 0}, l_{i_{1}, 0}\right)\left(\varepsilon+\varepsilon^{2}\right) \\
& \geq \beta_{i_{1}} u_{c}^{i_{1}}\left(\frac{\alpha}{\gamma}, 1\right) \alpha \varepsilon-u_{c}^{i_{1}}\left(c_{i_{1}, 0}, l_{i_{1}, 0}\right)\left(\varepsilon+\varepsilon^{2}\right) \\
& =\beta_{i_{1}} u_{c}^{i_{1}}\left(c_{i_{1}, 0}, 1\right) \frac{\varepsilon^{2}+\varepsilon}{\beta_{i_{1}}}-u_{c}^{i_{1}}\left(c_{i_{1}, 0}, l_{i_{1}, 0}\right)\left(\varepsilon+\varepsilon^{2}\right) \\
& =\left(\varepsilon^{2}+\varepsilon\right)\left[u_{c}^{i_{1}}\left(c_{i_{1}, 0}, 1\right)-u_{c}^{i_{1}}\left(c_{i_{1}, 0}, l_{i_{1}, 0}\right)\right] \geq 0
\end{aligned}
$$

If $u_{c l}^{i_{1}} \leq 0$, then

$$
\begin{aligned}
\frac{\Delta_{i_{1}}(\varepsilon)}{\lambda_{i_{1}}} & \geq \beta_{i_{1}} u^{i_{1}}(\alpha \varepsilon, \gamma \varepsilon)-u_{c}^{i_{1}}\left(c_{i_{1}, 0}, l_{i_{1}, 0}\right)\left(\varepsilon+\varepsilon^{2}\right) \\
& \geq \beta_{i_{1}} u_{c}^{i_{1}}(\alpha \varepsilon, \gamma \varepsilon) \alpha \varepsilon-u_{c}^{i_{1}}\left(c_{i_{1}, 0}, l_{i_{1}, 0}\right)\left(\varepsilon+\varepsilon^{2}\right) \\
& \geq\left(\varepsilon^{2}+\varepsilon\right)\left[u_{c}^{i_{1}}(\alpha \varepsilon, 1)-u_{c}^{i_{1}}\left(c_{i_{1}, 0}, l_{i_{1}, 0}\right)\right]
\end{aligned}
$$

Due to the Inada conditions on $u^{i_{1}}$, the term inside the bracket becomes nonnegative for $\varepsilon$ small enough.

We also have:

$$
\begin{aligned}
\frac{\Delta_{i_{2}}(\varepsilon)}{\lambda_{i_{2}}} & =\beta_{i_{2}}\left[u^{i_{2}}(\varepsilon, \varepsilon)-u^{i_{2}}\left(c_{i_{2}, 1}^{*}, l_{i_{2}, 1}^{*}\right)\right]+\left[u^{i_{2}}\left(c_{i_{2}, 0}, l_{i_{2}, 0}\right)-u^{i_{2}}\left(c_{i_{2}, 0}^{*}, l_{i_{2}, 0}^{*}\right)\right] \\
& \geq \beta_{i_{2}} u^{i_{2}}\left(\varepsilon^{2}, \varepsilon\right)+u_{c}^{i_{2}}\left(c_{i_{2}, 0}, l_{i_{2}, 0}\right) \varepsilon^{2} \geq 0
\end{aligned}
$$

Step 2: If $F(0, m)=0$, then by feasiblity $k_{2}^{*}=F(0, m)=0$. But this contradicts our claim in Step 1. We conclude that $k_{1}^{*}>0$. Consider next the case where $F(0, m)>0$. From Step 1 we know that $0 \leq k_{2}^{*}<f(0, m)$, so from Proposition 2(iv) it follows that $c_{i_{1}, 1}^{*}>0$ and $l_{i_{1}, 1}^{*}>0$. Observe that $L_{1}^{*}>0$ (if $L_{1}^{*}=0$ then by feasibility $c_{i_{1}, 1}^{*}=0:$ a contradiction). Consider the alternative feasible path $\left(\left(\mathbf{c}_{i}, \mathbf{l}_{i}\right)_{i}, \mathbf{k}\right)$ defined as follows:

i) $\quad c_{i_{1}, 0}=c_{i_{1}, 0}^{*}-\varepsilon, c_{i_{1}, 1}=c_{i_{1}, 1}^{*}+f\left(\varepsilon, L_{1}^{*}\right)-f\left(0, L_{1}^{*}\right), c_{i_{1}, t}=c_{i_{1}, t}^{*}, \forall t \geq 2$,

ii) $c_{i, t}=c_{i, t}^{*} \forall i \neq i_{1}, \forall t$ and $l_{i, t}=l_{i, t}^{*}, \forall i, \forall t$

iii) $k_{1}=\varepsilon, k_{t}=k_{t}^{*}, \forall t \geq 2$.

Define:

$$
\Delta_{\varepsilon}=\sum_{i \in I} \lambda_{i} \sum_{t=0}^{\infty} \beta_{i}^{t} u^{i}\left(c_{i, t}, l_{i, t}\right)-\sum_{i \in I} \lambda_{i} \sum_{t=0}^{\infty} \beta_{i}^{t} u^{i}\left(c_{i, t}^{*}, l_{i, t}^{*}\right)
$$


The concavity of $u^{i_{1}}$ and $f$ implies that

$$
\begin{aligned}
\frac{\Delta_{i_{1}}(\varepsilon)}{\lambda_{i_{1}}} & =u^{i_{1}}\left(c_{i_{1}, 0}, l_{i_{1}, 0}\right)-u^{i_{1}}\left(c_{i_{1}, 0}^{*}, l_{i_{1}, 0}^{*}\right)+\beta_{i_{1}}\left[u^{i_{1}}\left(c_{i_{1}, 1}, l_{i_{1}, 1}\right)-u^{i_{1}}\left(c_{i_{1}, 1}^{*}, l_{i_{1}, 1}^{*}\right)\right] \\
& \geq u_{c}^{i_{1}}\left(c_{i_{1}, 0}, l_{i_{1}, 0}\right)\left(c_{i_{1}, 0}-c_{i_{1}, 0}^{*}\right)+\beta_{i_{1}} u_{c}^{i_{1}}\left(c_{i_{1}, 1}, l_{i_{1}, 1}\right)\left[f\left(\varepsilon, L_{1}^{*}\right)-f\left(0, L_{1}^{*}\right)\right] \\
& \geq-u_{c}^{i_{1}}\left(c_{i_{1}, 0}, l_{i_{1}, 0}\right) \varepsilon+\beta_{i_{1}} u_{c}^{i_{1}}\left(c_{i_{1}, 1}, l_{i_{1}, 1}\right) f_{k}\left(\varepsilon, L_{1}^{*}\right) \varepsilon \\
& \geq \varepsilon\left[\beta_{i_{1}} u_{c}^{i_{1}}\left(c_{i_{1}, 1}, l_{i_{1}, 1}\right) f_{k}\left(\varepsilon, L_{1}^{*}\right)-u_{c}^{i_{1}}\left(c_{i_{1}, 0}, l_{i_{1}, 0}\right)\right]
\end{aligned}
$$

As $\varepsilon \rightarrow 0, u_{c}^{i_{1}}\left(c_{i_{1}, 1}, l_{i_{1}, 1}\right) f_{k}\left(\varepsilon, L_{1}^{*}\right) \rightarrow+\infty$ while $u_{c}^{i_{1}}\left(c_{i_{1}, 0}, l_{i_{1}, 0}\right) \rightarrow u_{c}^{i_{1}}\left(c_{i_{1}, 0}^{*}, l_{i_{1}, 0}^{*}\right)<$ $+\infty$. Hence, for $\varepsilon>0$ small enough, $\Delta_{i_{1}}(\varepsilon)>0:$ a contradiction. It follows that $k_{1}^{*}>0$. Working by induction we can show that $k_{t}^{*}>0$ for any $t$.

Proof of Lemma 5: Assume the contrary: $k_{0}>0, \mathbf{k}^{*}$ is optimal from $k_{0}$ and $k_{t}^{*} \rightarrow 0$. Since $f_{k}(0,1)>1$, for $k$ small enough, we have that $f(k, m)>k$. This implies that there exists a date $T$, such that, $0<k_{t+1}^{*}<f\left(k_{t}^{*}, m\right), \forall t \geq T$. We know that with any optimal solution $\mathbf{k}^{*}$ of problem $(Q)$, there exist associated sequences $\left(\mathbf{c}_{i}^{*}, \mathbf{l}_{i}^{*}\right)_{i \in I}$ for consumption and leisure, such that, $\left(\left(\mathbf{c}_{i}^{*}, \mathbf{l}_{i}^{*}\right)_{i \in I}, \mathbf{k}^{*}\right)$ is a solution to problem $(P)$. Recall that $L_{t}^{*}=m-\sum_{i \in I} l_{i, t}^{*}$. The proof follows in two steps:

Step 1: We claim that the sequence $\left(\frac{k_{t}^{*}}{L_{t}^{*}}\right)$ converges to zero.

Let $\left(\frac{k_{t_{n}}^{*}}{L_{t_{n}}^{*}}\right)$ be a subsequence such that:

$$
\limsup \sup _{t} \frac{k_{t}^{*}}{L_{t}^{*}}=\lim _{n} \frac{k_{t_{n}}^{*}}{L_{t_{n}}^{*}}
$$

Without loss of generality $\left(t_{n}\right)$ can be chosen such that $0<k_{t_{n}+1}^{*}<f\left(k_{t_{n}}^{*}, m\right)$ for any $n \in \mathbb{N}$ and $l_{i, t_{n}}^{*} \rightarrow l_{i} \in[0,1]$ for any $i \in I$. Moreover, assume that $\# I=m$ (if $\# I<m$ the claim follows directly). If $l_{i}<1$ for some $i$, it follows that $L_{t_{n}}^{*} \rightarrow L>0$ and the claim is true. Consider next the case where $l_{i}=1$ for all $i \in I$. In this case, $L_{t_{n}}^{*} \rightarrow 0$. The first order conditions for problem $(P)$ imply that for any $i \in I$, for any $n \in \mathbb{N}$ :

$$
F_{L}\left(k_{t_{n}}^{*}, L_{t_{n}}^{*}\right) \leq \frac{u_{l}^{i}\left(c_{i, t_{n}}^{*}, l_{i, t_{n}}^{*}\right)}{u_{c}^{i}\left(c_{i, t_{n}}^{*}, l_{i, t_{n}}^{*}\right)} .
$$

Define $\xi(x)=F(x, 1)-x F_{k}(x, 1)$. Since $f$ is homogeneous of degree $\alpha \in(0,1]$ we have:

$$
\begin{aligned}
F_{L}\left(k_{t_{n}}^{*}, L_{t_{n}}^{*}\right) & =\alpha\left(L_{t_{n}}^{*}\right)^{\alpha-1} \xi\left(\frac{k_{t_{n}}^{*}}{L_{t_{n}}^{*}}\right) \\
& \leq \frac{u_{l}^{i}\left(c_{i, t_{n}}^{*}, l_{i, t_{n}}^{*}\right)}{u_{c}^{i}\left(c_{i, t_{n}}^{*}, l_{i, t_{n}}^{*}\right)}
\end{aligned}
$$


Denote $B\left(k_{0}\right):=f\left(A\left(k_{0}\right), m\right)$. Since $l_{i, t_{n}}^{*} \rightarrow 1$ there must exist some $\varepsilon \in(0,1)$ and $N \in \mathbb{N}$ such that $1-\varepsilon \leq l_{t_{n}}^{*}, \forall n \geq N$. It follows that $\forall n \geq N$

$$
\begin{aligned}
\frac{u_{l}^{i}\left(c_{i, t_{n}}^{*}, l_{i, t_{n}}^{*}\right)}{u_{c}^{i}\left(c_{i, t_{n}}^{*}, l_{i, t_{n}}^{*}\right)} & \leq \frac{u^{i}\left(c_{i, t_{n}}^{*}, l_{i, t_{n}}^{*}\right)}{(1-\varepsilon) u_{c}^{i}\left(c_{i, t_{n}}^{*}, l_{i, t_{n}}^{*}\right)} \\
& \leq\left\{\begin{array}{c}
\frac{u^{i}\left(B\left(k_{0}\right), 1\right)}{(1-\varepsilon) u_{c}^{i}\left(B\left(k_{0}\right), 1-\varepsilon\right)}, \text { if } u_{12}^{i}>0 \\
\frac{\left.u^{i}\left(B k_{0}\right), 1\right)}{(1-\varepsilon) u_{c}^{i}\left(B\left(k_{0}\right), 1\right)}, \text { if } u_{12}^{i} \leq 0
\end{array}\right.
\end{aligned}
$$

Consider first the case where $\alpha \in(0,1)$. Combining (1) and (2) we get:

$$
\lim _{n} \alpha\left(L_{t_{n}}^{*}\right)^{\alpha-1} \xi\left(\frac{k_{t_{n}}^{*}}{L_{t_{n}}^{*}}\right) \leq M_{0} .
$$

For this to be true we must have:

$$
\xi\left(\lim _{n} \frac{k_{t_{n}}^{*}}{L_{t_{n}}^{*}}\right)=0 .
$$

Observe that $\xi$ is strictly increasing on $\mathbb{R}_{++}$. From the definition of $\xi$ and the concavity of $F$ we also have that $\xi(z)=F(z, 1)-z F_{k}(z, 1) \geq 0$ for any $z>0$. It follows that $\frac{k_{t_{n}}^{*}}{L_{t_{n}}^{*}} \rightarrow 0$.

Consider next the case where $\alpha=1$. Assume that $\frac{k_{t_{n}}^{*}}{L_{t_{n}}^{*}} \rightarrow z>0$. Since $f\left(k_{t}^{*}, L_{t}^{*}\right)=k_{t}^{*} f\left(1, \frac{L_{t}^{*}}{k_{t}^{*}}\right)$ and $k_{t}^{*} \rightarrow 0$, by feasibility it follows that $c_{i, t}^{*} \rightarrow 0$ for any $i$. Then combining (1) and (2) and using the Inada conditions on $u^{i}$ we arrive at a contradiction. We subsequently conclude that $\frac{k_{t_{n}}^{*}}{L_{t_{n}}^{*}} \rightarrow 0$.

Step 2: Choose some $\varepsilon>0$, such that, $f_{k}(\varepsilon, 1)>1$. Since $\frac{k_{t}^{*}}{L_{t}^{*}} \rightarrow 0$, there exists some date $T^{\prime}$, such that $\frac{k_{t}^{*}}{L_{t}^{*}} \leq \varepsilon, \forall t \geq T^{\prime}$.

For any $t \geq T_{1}=\max \left\{T, T^{\prime}\right\}$ Euler's equations hold, i.e for any $i \in I$, we have:

$$
u_{c}^{i}\left(c_{i, t}^{*}, l_{i, t}^{*}\right)=\beta_{i} u_{c}^{i}\left(c_{i, t+1}^{*}, l_{i, t+1}^{*}\right) f_{k}\left(k_{t+1}^{*}, L_{t+1}^{*}\right)
$$

It is easy to check that $f_{k}\left(k_{t+1}^{*}, L_{t+1}^{*}\right) \geq f_{k}(\varepsilon, 1), \forall t \geq T_{1}$. Indeed, if $L_{t+1}^{*} \geq 1$ the inequality is true since $f_{k L} \geq 0$. If $L_{t+1}^{*}<1$ observe that:

$$
\begin{aligned}
f_{k}\left(k_{t+1}^{*}, L_{t+1}^{*}\right) & =\left(L_{t+1}^{*}\right)^{\alpha-1} F_{k}\left(\frac{k_{t+1}^{*}}{L_{t+1}^{*}}, 1\right)+(1-\delta) \\
& \geq F_{k}\left(\frac{k_{t+1}^{*}}{L_{t+1}^{*}}, 1\right)+(1-\delta) \\
& \geq f_{k}(\varepsilon, 1) .
\end{aligned}
$$


It follows that there exists $T_{2} \geq T_{1}$ such that for any $t \geq T_{2}$ :

$$
\begin{aligned}
+\infty>u_{c}^{i}\left(c_{i, T_{2}}^{*}, l_{i, T_{2}}^{*}\right) & \geq u_{c}^{i}\left(c_{i, t}^{*}, l_{i, t}^{*}\right) \prod_{\tau=1}^{t-T_{2}}\left[\left(\min _{i} \beta_{i}\right) f_{k}\left(k_{t+\tau}^{*}, L_{t+\tau}^{*}\right)\right] \\
& \geq u_{c}^{i}\left(c_{i, t}^{*}, l_{i, t}^{*}\right) \prod_{\tau=1}^{t-T_{2}}\left[\left(\min _{i} \beta_{i}\right) f_{k}(\varepsilon, 1)\right] \\
& \geq A^{t-T_{2}} u_{c}^{i}\left(c_{i, t}^{*}, l_{i, t}^{*}\right)
\end{aligned}
$$

with $A=\left(\min _{i} \beta_{i}\right) f_{k}(\varepsilon, 1)>1$. Fix some $i \in I$. We distinguish two cases:

Case 1: Assume that $u_{c l}^{i} \leq 0$.

In this case, $\forall t>T_{2}$ we have:

$$
+\infty>u_{c}^{i}\left(c_{i, T_{2}}^{*}, l_{i, T_{2}}^{*}\right) \geq A^{t-T_{2}} u_{c}^{i}\left(c_{i, t}^{*}, l_{i, t}^{*}\right) \geq A^{t-T_{2}} u_{c}^{i}\left(B\left(k_{0}\right), 1\right) .
$$

Since $A^{t-T_{2}} \rightarrow+\infty$ as $t \rightarrow+\infty$, we obtain a contradiction.

Case 2: Assume that $u_{c l}^{i}>0$.

In this case, $\forall t>T_{2}$ we have:

$$
+\infty>u_{c}^{i}\left(c_{i, T_{2}}^{*}, l_{i, T_{2}}^{*}\right) \geq A^{t-T_{2}} u_{c}^{i}\left(c_{i, t}^{*}, l_{i, t}^{*}\right) .
$$

Since $A^{t-T_{2}} \rightarrow+\infty$ as $t \rightarrow+\infty$, it follows that $u_{c}^{i}\left(c_{i, t}^{*}, l_{i, t}^{*}\right) \rightarrow 0$. But $u_{c}^{i}\left(c_{i, t}^{*}, l_{i, t}^{*}\right) \geq$ $u_{c}^{i}\left(B\left(k_{0}\right), l_{i, t}^{*}\right)$ in which case $l_{i, t}^{*} \rightarrow 0$. Observe also that $\forall t>T_{2}$ we have:

$$
\begin{aligned}
u_{l}^{i}\left(c_{i, t}^{*}, l_{i, t}^{*}\right) & =u_{c}^{i}\left(c_{i, t}^{*}, l_{i, t}^{*}\right) f_{L}\left(k_{t}^{*}, L_{t}^{*}\right) \\
& \leq u_{c}^{i}\left(c_{i, t}^{*}, l_{i, t}^{*}\right) f\left(\frac{k_{t}^{*}}{L_{t}^{*}}, 1\right) \\
& \leq u_{c}^{i}\left(c_{i, t}^{*}, l_{i, t}^{*}\right) f(\varepsilon, 1) .
\end{aligned}
$$

It follows that $u_{l}^{i}\left(c_{i, t}^{*}, l_{i, t}^{*}\right) \rightarrow 0$ and therefore $c_{i, t}^{*} \rightarrow 0$.

By assumption $u^{i}(0,0)=0$. We have to distinguish three subcases.

i) Assume first that there exists $c>0$ such that $u^{i}(c, 0)>0$.

In this case, there exists $\widehat{c}>0$ such that $u_{c}^{i}(\widehat{c}, 0)>0$ (if not, it follows that $u_{c}^{i}(c, 0)=0, \forall c>0:$ a contradiction). Since $c_{i, t}^{*} \rightarrow 0, T_{2}$ can be chosen such that, for any $t>T_{2}$, we have $c_{i, t}^{*}<\widehat{c}$. In this case:

$$
+\infty>u_{c}^{i}\left(c_{i, T_{2}}^{*}, l_{i, T_{2}}^{*}\right) \geq A^{t-T_{2}} u_{c}^{i}\left(c_{i, t}^{*}, l_{i, t}^{*}\right) \geq A^{t-T_{2}} u_{c}^{i}(\widehat{c}, 0) .
$$

Since $A^{t-T_{2}} \rightarrow+\infty$ as $t \rightarrow+\infty$, we obtain a contradiction.

ii) Consider next the case where there exists $l>0$ such that $u^{i}(0, l)>0$. A similar argument implies that there exists $\widehat{l}$ such that $u_{l}^{i}(0, \widehat{l})>0$. Since $l_{i, t}^{*} \rightarrow 0, T_{2}$ can be chosen such that, for any $t>T_{2}$, we have $l_{i, t}^{*}<\widehat{l}$. In this case:

$$
+\infty>u_{l}^{i}\left(c_{i, t}^{*}, l_{i, t}^{*}\right) \geq u_{l}^{i}(0, \widehat{l})>0 .
$$


Since $u_{l}^{i}\left(c_{i, t}^{*}, l_{i, t}^{*}\right) \rightarrow 0$ as $t \rightarrow+\infty$, we obtain a contradiction.

iii) Consider finally the case where $u^{i}(c, 0)=u^{i}(0, l)=0$.

We know that $c_{i, t}^{*} \rightarrow 0$ and $l_{i, t}^{*} \rightarrow 0$. Observe that for any subsequence $\left(c_{i, t}^{*}, l_{i, t}^{*}\right)$ such that $c_{i, t}^{*} \leq l_{i, t}^{*}$, Assumption U5 implies that $u_{c}^{i}\left(c_{i, t}^{*}, l_{i, t}^{*}\right) \geq u_{c}^{i}\left(l_{i, t}^{*}, l_{i, t}^{*}\right) \geq$ $u_{c}^{i}(1,1)>0$ : a contradiction since we know that $u_{c}^{i}\left(c_{i, t}^{*}, l_{i, t}^{*}\right) \rightarrow 0$. In a similar way, for any subsequence $\left(c_{i, t}^{*}, l_{i, t}^{*}\right)_{t}$ such that $c_{i, t}^{*}>l_{i, t}^{*}$, Assumption U5 implies that $u_{l}^{i}\left(c_{i, t}^{*}, l_{i, t}^{*}\right) \geq u_{l}^{i}\left(c_{i, t}^{*}, c_{i, t}^{*}\right) \geq u_{l}^{i}\left(B\left(k_{0}\right), B\left(k_{0}\right)\right)>0$ : a contradiction since we know that $u_{l}^{i}\left(c_{i, t}^{*}, l_{i, t}^{*}\right) \rightarrow 0$.

\section{Appendix B: Uniqueness of steady state}

Given any $\nu \in(0,1]$ denote:

$$
\tilde{f}\left(\nu, k,\left(1-l_{i}\right)_{i \in I_{1}}\right):=f\left(k, m-\sum_{i \in I_{1}} l_{i}\right)+\nu \sum_{i \in I_{1}}\left(1-l_{i}\right)^{\alpha}+\nu\left(m-\# I_{1}\right) .
$$

Let $\underset{\sim}{0}=(0)_{i \in I_{2}}$ denote the zero vector in $\mathbb{R}^{\# I_{2}}$. Observe that

$$
\begin{aligned}
& \widehat{V}(\nu, \lambda, k, y):=\widetilde{V}(\nu, \lambda, \underset{\sim}{\underset{\sim}{0}, k, y)} \\
& =\max \sum_{i \in I_{1}} \lambda_{i} u^{i}\left(c_{i}, l_{i}\right) \\
& \text { s.t. } \sum_{i \in I_{1}} c_{i}+y \leq \tilde{f}\left(\nu, k,\left(1-l_{i}\right)_{i \in I_{1}}\right) \\
& c_{i} \geq 0, l_{i} \in[0,1], \forall i \in I_{1}
\end{aligned}
$$

is the indirect utility function associated with the Pareto optimal problem involving only the most patient consumers in an economy with production possibilities described by the function $\tilde{f}\left(\nu, k,\left(1-l_{i}\right)_{i \in I_{1}}\right)$. The corresponding value function is as follows:

$$
\begin{aligned}
\widehat{W}\left(\nu, k_{0}\right):= & \widetilde{W}\left(\nu, \underset{\sim}{0}, k_{0}\right) \\
= & \max \sum_{t=0}^{\infty} \beta^{t} \widehat{V}\left(\nu, \lambda, k_{t}, k_{t+1}\right) \\
& \text { s.t. } 0 \leq k_{t+1} \leq \widetilde{f}\left(\nu, k_{t}, m\right), \forall t \\
& k_{0} \geq 0, \text { is given. }
\end{aligned}
$$

The optimal policy function for this problem is given by:

$$
\widetilde{\varphi}(\nu, \underset{\sim}{0}, k)=\arg \max \{\widehat{V}(\nu, \lambda, k, y)+\beta \widehat{W}(\nu, y): 0 \leq y \leq \widetilde{f}(\nu, k, m)\}
$$


Let $k_{0}>0$ and $\mathbf{k}^{*}(\nu)$ be the optimal solution from $k_{0}$ to problem $\widehat{Q}(\nu)$. In this case, we write $k_{t+1}^{*}(\nu)=\widetilde{\varphi}\left(\nu, \underset{\sim}{0}, k_{t}^{*}(\nu)\right)=\widetilde{\varphi}^{t}\left(\nu, \underset{\sim}{0}, k_{0}\right)$. The argument of the proof is as follows. We first show that given any $\nu \in(0,1]$ the problem $\widehat{Q}(\nu)$ has a unique steady state denoted $k^{s}(\nu)$. We subsequently prove that $k^{s}(\nu)$ converges to some $k^{s}$ as $\nu \rightarrow 0$. The last step involves to show that, independent of the initial capital stock $k_{0}$, the optimal path associated with problem $\widehat{Q}$ converges to $k^{s}$. Working in this direction we need the following two results:

Claim 1 Fix some $\nu \in(0,1]$. Let $k_{0}>0$ and $\mathbf{k}^{*}(\nu)$ be a solution from $k_{0}$ to problem $\widehat{Q}(\nu)$. Then, $\left(k_{t}^{*}(\nu)\right)$ converges monotonically to a unique steady state.

Proof: Fix some $\nu \in[0,1]$. It is easy to check that $\left.\widetilde{f} \nu, k,\left(1-l_{i}\right)_{i \in I_{1}}\right)$ satisfies the assumptions F1-F5. Proposition 8 shows that $\widehat{V}(\nu, \lambda, k, y)$ is supermodular in the interior of $\widetilde{D}(\nu)$. This implies that $\left(k_{t}^{*}(\nu)\right)$ is monotonic. The method of proof in Lemma 4 and 5 can apply to show that $\left(k_{t}^{*}(\nu)\right)$ is strictly positive and does not converge to zero. It then follows that $\left(k_{t}^{*}(\nu)\right)$ converges to some $k^{s}(\nu)>0$. We next show that $k^{s}(\nu)$ is in addition unique.

Assume that there are more than one nontrivial steady states. Their number must be finite ${ }^{4}$. The roots of the Jacobian evaluated at any steady state will be the solution of:

$$
\beta \lambda^{2} \widehat{V}_{12}+\left[\widehat{V}_{22}+\beta \widehat{V}_{11}\right] \lambda+\widehat{V}_{12}=0
$$

A sufficient condition for saddle point stability ${ }^{5}$ is:

$$
-(1+\beta) \widehat{V}_{12}+\beta \widehat{V}_{11}+\widehat{V}_{22}<0
$$

We know that $\widehat{V}_{12}>0$. Since $\widehat{V}$ is concave, we also have that $\widehat{V}_{11}, \widehat{V}_{22} \leq 0$. Since the number of steady states is finite one can find $\underline{k}^{s}(\nu), \bar{k}^{s}(\nu)$, with $\underline{k}^{s}(\nu)<$ $\bar{k}^{s}(\nu)$, such that, no point $k \in\left(\underline{k}^{s}(\nu), \bar{k}^{s}(\nu)\right)$ is a steady state. Saddle point stability implies that there exists $\underline{\varepsilon}>0$, such that, for any $k_{0} \in\left(\underline{k}^{s}(\nu)-\right.$ $\left.\underline{\varepsilon}, \underline{k}^{s}(\nu)+\underline{\varepsilon}\right), \widetilde{\varphi}^{t}\left(\nu, \underset{\sim}{0}, k_{0}\right) \rightarrow \underline{k}^{s}(\nu)$. Similarly, there must exist $\bar{\varepsilon}$, such that, for any $k_{0} \in\left(\bar{k}^{s}(\nu)-\bar{\varepsilon}, \bar{k}^{s}(\nu)+\bar{\varepsilon}\right), \widetilde{\varphi}^{t}\left(\nu, \underset{\sim}{0}, k_{0}\right) \rightarrow \bar{k}^{s}(\nu)$. Let $\underline{k}=\sup _{\underline{\varepsilon}}\left(\underline{k}^{s}(\nu)+\underline{\varepsilon}\right)$ and $\bar{k}=\inf _{\bar{\varepsilon}}\left(\bar{k}^{s}(\nu)-\bar{\varepsilon}\right)$. Monotonicity implies that

$$
\underline{k}^{s}(\nu)<\underline{k}=k=\bar{k}<\bar{k}^{s}(\nu) .
$$

For any $\bar{k}^{s}(\nu)>k^{\prime}>k$, we have $\widetilde{\varphi}\left(\nu, \underset{\sim}{0}, k^{\prime}\right)>k^{\prime}$. The continuity of $\widetilde{\varphi}$ implies that $\widetilde{\varphi}(\nu, \underset{\sim}{0}, k) \geq k$. In a similar way, for any $\underline{k}^{s}(\nu)<k^{\prime}<k$, we have $\widetilde{\varphi}\left(\nu, \underset{\sim}{0}, k^{\prime}\right)<k^{\prime}$,

\footnotetext{
${ }^{4}$ Assume that the set of nontrivial steady states is infinite. Since this set is compact, there will be a subsequence $\left(k_{n}^{s}(\nu)\right)$ that converges to some $k^{s}(\nu)$. But this leads to a contradiction, since by the Implicit Function Theorem, for $k^{s}(\nu)$ there is an open neighborhood $\mathcal{U}\left(k^{s}(\nu)\right)$ where $k^{s}(\nu)$ is the unique solution to the steady state Euler equations.

${ }^{5}$ Refer to Majumdar et al. (2000) Ch. 2, Section 7.2.
} 
and by continuity, $\widetilde{\varphi}(\nu, \underset{\sim}{0}, k) \leq k$. But this implies that $k$ is a steady state: a contradiction. Hence, $k^{s}(\nu)$ is uniquely determined.

Claim 2 Let $k_{0}>0$. For any $\varepsilon>0$, there exists $T(\varepsilon)$ such that for any $\nu \in(0,1]$, for any $t \geq T(\varepsilon)$ we have:

$$
\left|\widetilde{\varphi}^{t}\left(\nu, \underset{\sim}{0}, k_{0}\right)-k^{s}(\nu)\right|<\varepsilon
$$

Proof: We know that $\widetilde{\varphi}^{t}\left(\nu, \underset{\sim}{0}, k_{0}\right) \rightarrow k^{s}(\nu)$. This implies that for any $\varepsilon>0$, there exists $T(\nu, \varepsilon)$ such that:

$$
\left|\widetilde{\varphi}^{T(\nu, \varepsilon)}\left(\nu, \underset{\sim}{0}, k_{0}\right)-k^{s}(\nu)\right|<\varepsilon .
$$

The continuity of $\widetilde{\varphi}$ implies that there exists a neighborhood $\mathcal{N}(\nu)$ of $\nu$, such that, for any $\nu^{\prime} \in \mathcal{N}(\nu)$ we have:

$$
\left|\widetilde{\varphi}^{T\left(\nu^{\prime}, \varepsilon\right)}\left(\nu^{\prime}, \underset{\sim}{0}, k_{0}\right)-k^{s}\left(\nu^{\prime}\right)\right|<\varepsilon .
$$

Take a finite covering $\left(\mathcal{N}\left(\nu_{j}\right)\right)_{j=1}^{J}$ of $[0,1]$ and let $T(\varepsilon)=\max _{j \in\{1, \ldots, J\}}\left\{T\left(\nu_{j}, \varepsilon\right)\right\}$.

Fix some $k_{0}>0$. Let $\left(\nu_{n}\right)$ be a sequence such that $\nu_{n} \rightarrow 0$. Consider the sequence of optimal paths $\left(\mathbf{k}^{*}\left(\nu_{n}\right)\right)$ associated to the sequence of intertemporal problems $\left(Q\left(\nu_{n}\right)\right)$ having an initial capital stock equal to $k_{0}$. Assume that $k_{0} \leq$ $k^{s}\left(\nu_{n}\right)$ for any $n \in \mathbb{N}$. Recall that $k_{t}^{*}\left(\nu_{n}\right)=\widetilde{\varphi}^{t}\left(\nu_{n}, \underset{\sim}{0}, k_{0}\right)$ and $k_{t}^{*}=\varphi^{t}\left(\underset{\sim}{0}, k_{0}\right)$. We have:

$$
k_{0} \leq \widetilde{\varphi}\left(\nu_{n}, \underset{\sim}{0}, k_{0}\right) \leq \ldots \leq \widetilde{\varphi}^{t}\left(\nu_{n}, \underset{\sim}{0}, k_{0}\right) \leq \ldots \leq k^{s}\left(\nu_{n}\right)
$$

Denote $\underset{\sim}{k}:=\liminf _{n} k^{s}\left(\nu_{n}\right)$. The continuity of $\widetilde{\varphi}^{t}\left(\nu, \underset{\sim}{0}, k_{0}\right)$ with respect to $\nu$ implies that $\widetilde{\varphi}^{t}\left(\nu_{n}, \underset{\sim}{0}, k_{0}\right) \rightarrow \varphi^{t}\left(\underset{\sim}{0}, k_{0}^{*}\right)$ as $\nu_{n} \rightarrow 0$. We have:

$$
k_{0} \leq \varphi\left(\underset{\sim}{0}, k_{0}^{*}\right) \leq \ldots \leq \varphi^{t}\left(\underset{\sim}{0}, k_{0}^{*}\right) \leq \ldots \leq \underset{\sim}{k}
$$

Assume that $k_{t}^{*} \rightarrow k<\underset{\sim}{k}$. In this case there exists some $N$ and an $\varepsilon>0$ such that $k+\varepsilon<k^{s}\left(\nu_{n}\right)$ for any $n \geq N$. From Claim 2, it follows that there exists some $T(\varepsilon)$ independent of $\left(\nu_{n}\right)_{n \geq N}$ such that

$$
k+\varepsilon<k_{T(\varepsilon)}^{*}\left(\nu_{n}\right)<k^{s}\left(\nu_{n}\right) .
$$

Letting $\nu_{n} \rightarrow 0$ we get

$$
k+\varepsilon<k_{T(\varepsilon)}^{*} \leq k_{T(\varepsilon)+1}^{*} \leq \ldots \leq k,
$$




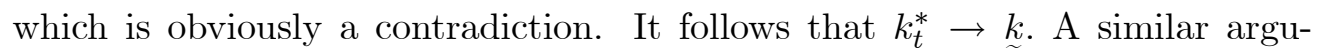

ment can apply to show that $k_{t}^{*} \rightarrow \widetilde{k}$ where $\widetilde{k}:=\limsup _{n} k^{s}\left(\nu_{n}\right)$. As a result $\lim _{\nu_{n} \rightarrow 0} k^{s}\left(\nu_{n}\right)$ exists. Denote this limit by $k^{s}$. The same is true if we had assumed alternatively that $k_{0} \geq k^{s}\left(\nu_{n}\right)$ for any $n \in \mathbb{N}$. In either case $\lim _{\nu_{n} \rightarrow 0} k^{s}\left(\nu_{n}\right)=k^{s}$ and $k_{t}^{*} \rightarrow k^{s}$. We have proved that for any $k_{0}>0$ the optimal path $\left(k_{t}^{*}\right)$ converges to $k^{s}$ which is independent of $k_{0}$. The proof is complete.

\section{References}

[1] Becker A. R.: On the long-run steady state in a simple dynamic model of equilibrium with heterogeneous households, Quarterly Journal of Economics, 95, 375-383 (1980)

[2] Becker A. R. : An example of optimal growth with heterogeneous agents and twisted turnpike, Indiana University unpublished manuscript.(2005)

[3] Becker A. R. and Foias C.: A characterization of Ramsey equilibrium, Journal of Economic Theory, 41, 173-184 (1987)

[4] Becker A. R. and Foias C.: The local bifurcation of Ramsey equilibrium, Economic Theory, 4, 719-744 (1994)

[5] Bewley, T.F.: An integration of equilibrium theory and turnpike theory, Journal of Mathematical Economics, 10, 233-267 (1982)

[6] Markovian equilibrium in infinite horizon economies with incomplete markets and public policy

[7] Coles, J.: Equilibrium turnpike theory with constant returns to scale and possible heterogeneous discount factors, International Economic Review, 26, 671-679 (1985)

[8] M. Datta, L. J. Mirman, O. F. Morand and Reffett, K. L., Markovian equilibrium in infinite horizon economies with incomplete markets and public policy, Journal of Mathematical Economics, 41, 505-544, (2005)

[9] Duran, J. and Le Van, C.: A simple proof of existence of equilibrium in a one sector growth model with bounded or unbounded returns from below Macroeconomic Dynamics, (2003)

[10] Dechert, W.D. and Nishimura K.: A complete characterization of optimal growth paths in an aggregative model with a no-concave production function, Journal of Economic Theory, 31, 332-354 (1985) 
[11] Florenzano, M., Le Van, C. and Gourdel, P.: Finite Dimensional and Optimization, Studies in Economic Theory 13, Springer-Verlag 2001

[12] Le Van, C. and L. Morhaim (2002), "Optimal growth models with bounded or unbounded returns: A unifying approach", Journal of Economic Theory 105, 158-187

[13] C. Le Van, L. Morhaim and C.H. Dimaria , The discrete time version of Romer's AK model, Economic Theory 20, 133-158, (2002).

[14] Le Van, C. and Vailakis Y.: Existence of a competitive equilibrium in a onesector growth model with heterogeneous agents and irreversible investment, Economic Theory, 22, 743-771 (2003)

[15] Majumdar, Mukul, Mitra, T. and Nishimura K.: Optimization and chaos, Studies in Economic Theory 11, Springer-Verlag 2000.

[16] Mas-Colell, A., Whinston, M. and Green R.J.: Microeconomic Theory. Oxford University Press 1995

[17] Mitra, T.: On optimal economic growth with variable discount rates: Existence and stability results, International Economic Review, 20, 133-145 (1979)

[18] Sorger, G.: On the structure of Ramsey equilibrium: Cycles, indeterminacy and sunspots, Economic Theory, 4, 745-764 (1994)

[19] Stokey, N. and Lucas Jr., R.E. with Prescott, E.C.: Recursive Methods in Economic Dynamics. Harvard University Press 1989 